\title{
1 Distinct ensembles in the noradrenergic locus coeruleus evoke diverse cortical states
}

3 Shahryar Noei ${ }^{1,2}$, Ioannis S. Zouridis ${ }^{3,6}$, Nikos K. Logothetis ${ }^{6,7}$, Stefano Panzeri ${ }^{1,8,9}$, Nelson

4 K. Totah ${ }^{4,5,6,9} *$

$5 \quad{ }^{1}$ Neural Computation Laboratory, Istituto Italiano di Tecnologia, Rovereto, Italy

$6 \quad{ }^{2}$ Center for Mind/Brain Sciences, University of Trento, Rovereto, Italy

$7 \quad{ }^{3}$ Graduate Training Centre of Neuroscience, University of Tübingen, Tübingen, Germany

$8{ }^{4}$ Helsinki Institute of Life Science (HiLIFE), University of Helsinki, Helsinki, Finland

$9{ }^{5}$ Faculty of Pharmacy, University of Helsinki, Helsinki, Finland

$10{ }^{6}$ Dept. of Physiol. of Cog. Processes, MPI Biological Cybernetics, Tübingen, Germany

$11{ }^{7}$ Division of Imaging Sci. and Biomed. Eng., University of Manchester, Manchester, UK

$12{ }^{8}$ Department of Neural Information Processing, Center for Molecular Neurobiology (ZMNH),

13 University Medical Center Hamburg-Eppendorf (UKE), Hamburg, Germany

$14{ }^{9}$ Senior authors

$15 *$ Corresponding Author

16 Correspondence: nelson.totah@helsinki.fi 


\section{Abstract}

19 The noradrenergic locus coeruleus (LC) is a crucial controller of brain and behavioral states. 20 Activating LC neurons synchronously en masse by electrical or optogenetic stimulation

21 promotes a stereotypical "activated" high-frequency cortical state. However, it has been recently 22 reported that spontaneous LC cell-pairs have sparse yet structured time-averaged cross23 correlations, which is unlike the high synchrony of en masse neuronal stimulation. This suggests

24 the untested possibility that LC population activity may be made of distinct multi-cell ensembles 25 each with unique temporal evolution of activity. We used non-negative matrix factorization 26 (NMF) to analyze large populations of LC single units simultaneously recorded in the rat LC. 27 Synthetic spike train simulations showed that NMF, unlike the traditional time-averaged pairwise 28 correlations, detects both the precise neuronal composition and the activation time courses of 29 each ensemble. NMF identified the existence of robust ensembles of spontaneously co-active LC 30 neurons. Since LC neurons selectively project to specific forebrain regions, we hypothesized that 31 individual LC ensembles produce different cortical states. To test this hypothesis, we triggered 32 local field potentials (LFP) in cortical area 24a on the activation of distinct LC ensembles. We 33 found four cortical states, each with different spectro-temporal LFP characteristics, that were 34 robust across sessions and animals. While some LC ensembles triggered the activated state, 35 others were associated with a beta oscillation-specific state or a reduced high frequency 36 oscillation state. Thus - in contrast to the stereotypical "activated" brain state evoked by en 37 masse LC stimulation - spontaneous activation of distinct LC ensembles can control a multitude 38 of cortical states.

39 Keywords: ensemble, cortical state, gamma oscillation, beta oscillation, slow wave, 40 neuromodulation, locus coeruleus, norepinephrine, area $24 \mathrm{a}$, prelimbic cortex, anterior cingulate 41 cortex 


\section{Introduction}

44 Flexible behavior is associated with transitions across diverse cortical states. For example,

45 various states of wakefulness, perceptual ability, and behavioral activity are associated with

46 different cortical local field potential (LFP) and EEG states each with its own clear spectro-

47 temporal pattern of neural oscillations (1-3). Behavioral state transitions, such as waking from

48 sleep or entering a state of heightened stress and reacting more quickly to stimuli, are associated

49 with cortical state transition. These changes are not necessarily driven by external stimuli.

50 Instead, cortical state can be controlled by factors internal to the organism (e.g., sleep need,

51 perceived stress) and therefore arise from self-organized neuronal interactions. It remains unclear

52 exactly which interactions among neurons control cortical states.

53 Cortical states are mediated, at least in part, by the noradrenergic brainstem nucleus, locus

54 coeruleus (LC). The LC releases norepinephrine to modulate neuronal excitability (4-7).

55 Noradrenergic neuromodulation of cortical state has been studied using direct electrical or

56 optogenetic stimulation. Such stimulation evokes highly synchronous and en masse activation of

57 many LC neurons because this brainstem nucleus (in rats) contains $\sim 1,600$ neurons tightly

58 packed into a small volume of 200 x 500 x 1000 um $(8,9)$. For instance, a low current $(0.03$ -

$590.05 \mathrm{~mA}$ ) pulse evokes spiking up to $400 \mathrm{um}$ from stimulation site in rat LC (10). Thus, even

60 minimal levels of stimulation will synchronously activate many LC neurons given the small

61 dimensions of the nucleus. Such en masse LC population activation evokes a stereotypical

62 "activated" cortical state characterized by increased high-frequency oscillation power, regardless

63 of whether the subject is anesthetized or non-anesthetized (10-13). Critically, it is still unknown

64 how spontaneous population activity in the LC, as opposed to en masse LC activity evoked by

65 stimulation, relates to the control of cortical state.

66 Although modulation of cortical state by the LC has largely been studied using external artificial

67 stimulation of LC, cortical state emerges from spontaneously occurring, internal neuronal

68 interactions. The spontaneous activity of the LC neuronal population has been traditionally

69 thought to be highly synchronous (14-19), akin to the en masse neuronal population activity

70 evoked by LC stimulation. However, recent findings suggest that this standard view might not

71 describe spontaneous LC activity accurately. Graph-theoretic analysis of time-averaged cross- 
72 correlations among pairs of spontaneously active LC neurons in anesthetized rats has reported

73 sparse yet structured pairwise correlations that are unlike the high synchrony of en masse

74 stimulation (20). These recent findings suggest that LC population activity might potentially

75 consist of multi-cell ensembles that are activated at different times. Importantly, however, these

76 prior pairwise graph-theoretic analyses were based upon time-averaged measures (20) and were

77 therefore unable to detect multi-cell ensembles or to resolve ensemble activity over time. Here,

78 we used non-negative matrix factorization (NMF) to analyze large populations of simultaneously

79 recorded single units in the rat LC. Synthetic spike trains simulations showed that NMF, unlike

80 the traditionally used time-averaged pairwise correlations, detects both the precise neuronal

81 composition and the activation time courses of each ensemble. This allowed us to demonstrate

82 that LC population activity consists of discrete LC ensembles each with its own evolution of

83 activity over time.

84 Given that LC neurons selectively project to specific forebrain regions, we investigated how 85 individual LC ensembles produce different cortical states. One possibility is that different LC 86 ensembles may simply evoke the stereotypical activated state (the only cortical state associated 87 with LC neuromodulation of the forebrain), as observed in prior anesthetized and non88 anesthetized stimulation experiments, but with different LC ensembles being associated with 89 variations in cortical activated state duration or power magnitude. A second and more intriguing 90 possibility is that distinct LC ensembles evoke different cortical states, each with a different 91 spectral signature. This latter case would support a new perspective that LC ensembles may 92 provide a nuanced contribution to the broad set of cortical states that characterize flexible 93 behavior.

94 Since our methods allowed tracking the spontaneous temporal dynamics of individual LC 95 ensembles, we could relate ongoing LC ensemble dynamics to cortical state dynamics. By using 96 the time-dependent power of the simultaneously recorded LFP in cortical area 24a as an index of 97 cortical state, we performed LC ensemble-triggered LFP (LCET-LFP) analysis, which triggered 98 cortical state on the activation of each distinct LC ensemble $(21,22)$. In contrast to the standard 99 view that LC population activity evokes a stereotypical activated cortical state, we observed 100 heterogenous cortical states with different spectral and temporal properties that depended on 101 which LC ensemble was active. Importantly, when different ensembles were spontaneously 
102 coactivated (more similar to en masse synchrony of population activity), the associated cortical

103 states were more homogenous and were more similar to the stereotypical activated state resulting

104 from electrical or optogenetic stimulation. Our results detail spontaneously occurring brain-

105 internal neuronal dynamics that control cortical state by demonstrating that the spontaneous

106 structuring of LC population activity as a collection of discrete ensembles can evoke different

107 cortical states.

108 Results

109 We aimed first to establish whether spontaneous LC population activity was made of subsets of 110 coactive neurons (ensembles) activating spontaneously at different times and then, if so, to 111 characterize their relationship with ongoing cortical state using LCET-LFP. We recorded many 112 LC single units simultaneously (range: 5 to 34 units; average: 19 units; $\mathrm{N}=15$ male rats) using a 113 silicon probe with 32 electrodes confined to the core of the LC nucleus. Probe location was 114 verified histologically in coronal tissue sections. Neuronal identity was confirmed at the end of 115 the experiments using intra-peritoneal injection of the alpha-2 agonist, clonidine, which inhibited 116 spiking on all electrodes. Spikes recorded from outside the LC core would not have been 117 inhibited due to the lack of alpha-2 adrenergic receptors in nearby brain structures (21). We 118 simultaneously recorded cortical local field potential (8 kHz lowpass filtered) from cortical area 11924 a (anterior cingulate cortex) (22) using a tungsten electrode in 9 of the 15 rats.

120 Spontaneous LC population activity consists of distinct ensembles with independent temporal 121 dynamics

122 It is currently unknown whether spontaneous LC population activity is made of ensembles (i.e., 123 sub-sets of simultaneously coactive neurons) and how the activity of ensembles changes over 124 time. We assessed whether LC population activity consists of ensembles using non-negative 125 matrix factorization (NMF) on the population vector made of the spike counts of simultaneously 126 recorded single units, independently for each rat. To create the spike count population vectors, 127 we binned activity in sliding windows that were $100 \mathrm{msec}$ long, which is the time scale capturing 128 most of the synchrony among LC single unit pairs (20). Figure 1A illustrates how NMF works 129 on hypothetical single unit spiking data. NMF decomposes the matrix containing the population 
130 vectors at all time points as a sum of K non-negative spatial modules, each multiplied by a non131 negative activation coefficient. A spatial module may be thought of as a specific, often-recurring, 132 population firing pattern. Formally, it is a vector specifying the relative strength of firing of each 133 single unit within the population $(23,24)$. Thresholding these spatial module values defines the 134 specific single units that were significantly active within each module. On the other hand, the 135 activation coefficients of each spatial module at any given time describe how strongly the spatial 136 module is recruited at that time. We determined $\mathrm{K}$ for each rat based on two criteria. First, the 137 chosen $\mathrm{K}$ explained a high amount of variance in the data with the fewest possible number of 138 spatial modules, K. In other words, K was in the "elbow" region of the reconstruction error, 139 which when plotted as a function of the possible number of spatial modules, meant that a higher $140 \mathrm{~K}$ would have given diminishing returns in terms of data reconstruction accuracy. Second, the 141 selected value for K yielded a stable recovery of the spatial modules from the data regardless of 142 the random initialization of the decomposition optimization procedure (see Methods for 143 additional details and Figure S1). By thresholding the time course of activation coefficients to 144 distinguish the times of significant recruitment of each spatial module, we defined the times of 145 activation of each spatial module. The spatial modules will be referred to as "ensembles" and the 146 activation times of spatial modules will be referred to as "ensemble activation times." In order to 147 study the contribution of LC population activity to cortical state, in the spirit of prior work (25), 148 after using NMF to isolate LC ensembles we then performed a LCET-LFP analysis. In this 149 analysis, we triggered the cortical LFP on localized neuronal events - specifically the activation 150 of distinct LC ensembles (Figure 1A).

151 NMF revealed for the first time that LC population activity consists of multiple, discrete 152 ensembles of co-active neurons. We detected 146 ensembles from 15 rats. Figure 1B shows the 153 spike rasters of 24 simultaneously recorded single units around the time point at which a subset 154 of 7 single units co-activated as an ensemble. The rasters show that those single units which 155 belong to the ensemble increased their firing rate during ensemble activation, whereas other 156 single units not assigned to that ensemble maintained their ongoing pattern of activity without 157 systematic variations around the time of ensemble activation (at $\mathrm{t}=0 \mathrm{sec}$ ). Figure 1C shows 158 another example in which LC population activity was decomposed into 5 distinct ensembles. The 159 ensembles were active in most cases at different times, but occasionally more than one ensemble 
was simultaneously active (e.g., brown and red lines at $\mathrm{t}=0.5 \mathrm{sec}$ ). Reconstructing the total population firing rate as function of time through NMF decomposition (i.e., essentially summing up the activation time courses across the 5 ensembles) returned a close approximation of the pooled population spike rate (blue line in upper left panel). This example illustrates that LC neuronal population activity is not merely only sparsely correlated, as shown in prior work (20), but is actually made of a nuanced sequence of distinct ensembles activating at largely nonoverlapping times.

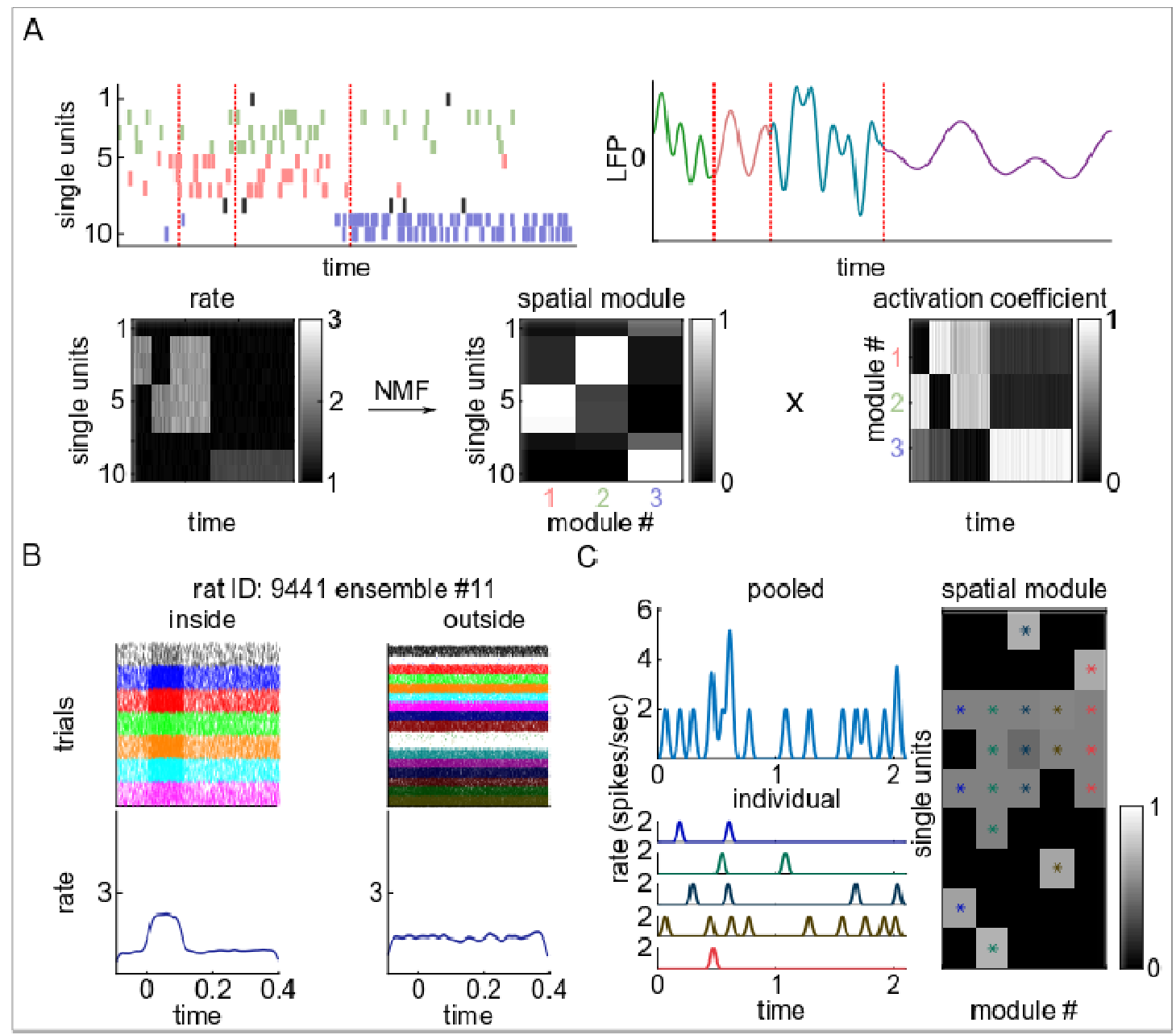

Figure 1. Spontaneous LC neuronal population activity consists of discrete ensembles. (A) An example using hypothetical data to illustrate how NMF detects ensembles and the time courses of ensemble activity. The spike rasters of 10 single units along with an illustration of cortical LFP is depicted in the top panel. The spikes of different single units, which co-activate as a group and therefore belong to various ensembles, are plotted in distinct colors. The different cortical LFP states are also plotted in various colors. The bottom left panel illustrates the spike rasters of the neuronal population as binned spike rate over time. Larger spike rates are in lighter 
color. The bottom right two plots show the outcome of NMF on the matrix of population spike rate. The population rate is decomposed as a sum of $\mathrm{K}$ spatial modules (in this example, there are 3 ). It is apparent from the spike rasters (top left) and their replotting as binned spike rates (lower left), that units 2, 3, and 4 often spike together in the first half of the time window (green spikes). Also, during this time window, units 5, 6, and 7 also spike together (red spikes). In the latter half of the window, units 9 and 10 tend to spike together (blue spikes). Therefore, there are 3 ensembles. Note that the first two ensembles are also co-activated briefly just prior to the third ensemble becoming active. The spatial modules (lower middle) resulting from NMF identify 3 specific reoccurring population firing patterns across single units. A threshold is applied to the values of the spatial modules to specify which single units are active in each population firing pattern. For example, module 2 (green) has high values for units 2, 3, and 4; thus, these units are co-active (i.e., ensemble 2). NMF also returns an activation coefficient (lower right) for each module, which represents the strength of recruitment of each module (i.e., each distinct population firing pattern) over time. When activation coefficients are high for a given module (lighter colors), that specific population firing pattern is occurring. Accordingly, NMF recapitulates the patterns observable in the spike raster (top left); ensemble 1 (units 2, 3, and 4, green spikes) and ensemble 2 (units 5, 6, and 7, red spikes) are active during the first half of the recording and are briefly co-active as an ensemble-pair before the latter half of the recording, during which ensemble 3 (units 9 and 10, blue spikes) is active. (B) The spike rasters and perievent time histograms (PETHs) are shown for one exemplar LC ensemble (actual data). The left panel shows spike rasters of the single units inside the ensemble aligned to the ensemble activation times $(t=0 \mathrm{sec})$. In these spike rasters, each ensemble activation event is a "trial." The PETHs of trial-averaged spike rate across all units in the ensemble are shown below the rasters. The right panel depicts the ensemble activation-triggered spiking of single units that were not assigned to that ensemble. The plot shows that units inside the ensemble increased their firing rate at ensemble activation times, whereas units not assigned to the ensemble did not change their firing rate in any systematic way. (C) An example from actual LC data in which NMF found 5 ensembles among 9 single units. The upper left panel plots the population activity (i.e., the summed spiking of all simultaneously recorded single units) over a 2 second epoch. The lower left panel plots the activation coefficients of the 5 ensembles. Each ensemble is plotted in a different color. The right panel plots the spatial module values. A threshold was applied to these values to specify which single units were significantly active in each spatial module (i.e., ensemble). Threshold crossings are marked with an asterisk with a different color for each ensemble.

Given that this is the first known demonstration of LC ensembles, we further characterized the spatial-temporal and cell type-specific properties of the ensembles. We first assessed the temporal dynamics of ensemble activations. Most ensembles were only transiently active for 100 msec on average. However, the duration of the inactive periods was highly-variable across ensembles (median \pm s.d. $=611 \pm 295 \mathrm{msec}$ ), such that ensembles were quiet for a wide variety of durations before being briefly active for approximately $100 \mathrm{msec}$ (Figure 2A). These findings 
216 suggest that distinct ensembles spontaneously activate at largely different times relative to one

217 another. The largely non-overlapping activation times of the ensembles will be systematically

218 demonstrated in a later section.

219 We found that ensembles were made of relatively small sub-sets of single units. On average, $22027 \%$ of single units were active in ensembles relative to the total number of simultaneously 221 recorded single units in each rat (Figure 2B). Ensemble size ranged from 6\% - 62\% of the 222 simultaneously recorded single units (Figure 2C). NMF allows single units to participate in 223 more than one ensemble. Therefore, we assessed whether ensembles consisted of totally separate 224 sets of single units or a more complicated structure of overlapping single units. We counted the 225 number of single units assigned to one ensemble, to multiple ensembles, or to no ensemble. Out 226 of 285 single units, 115 single units fired as part of multiple ensembles (40.4\%), 149 were active 227 in only a single ensemble (52.3\%), and the remaining 21 units did not participate in any 228 ensemble (Figure 2D). Although single units did spike in multiple ensembles, the probability 229 that a neuron took part in only one ensemble was higher than the probability that a neuron took 230 part in more than one ensemble (binomial test, $\mathrm{p}=0.04$ ).

231 Our recent work has shown that the LC contains two types of single units, termed "narrow" or 232 "wide" type, which are distinguishable by their extracellular waveform shape and have several 233 physiological and functional differences (20). We assessed (by random resampling) if the 234 proportion of each unit type participating in each ensemble was statistically different from what 235 would be expected if ensembles were formed by units taken randomly regardless of their type. 236 For all rats, the hypothesis that ensembles are formed by combining units regardless of their type 237 was rejected $(\mathrm{p}<0.05)$. Accordingly, ensembles preferentially consist of the same type of unit 238 (Figure 2E).

239 We found that ensembles were made of single units that were spatially diffuse. The location of 240 the maximal average action potential amplitude on the electrode array was used to assign a 241 spatial location to the single unit. We examined the distribution within each ensemble of the 242 distance among the electrodes from which each cell pair within an ensemble was recorded 243 (Figure 2F). There was a wide spread of distances between the units in each ensemble. 
244 Therefore, LC ensembles do not follow a topographical arrangement in the dorsal-ventral or

245 medio-lateral aspects of the core of the LC nucleus.

246 Finally, we assessed how firing strength varied across LC ensembles. In order to quantify the

247 firing strength of LC ensembles, we calculated the average spike rate of all single units within

248 the ensemble (when the ensemble was active) using peri-event time histograms (PETHs). Each

249 event was an ensemble activation time. The PETHs were calculated from $100 \mathrm{msec}$ before each

250 ensemble activation event until $400 \mathrm{msec}$ afterwards. We characterized the diversity of firing

251 strengths across ensembles by clustering the PETHs of 146 ensembles using Principal

252 Component Analysis and Gaussian Mixture Models. When visualizing the data in two

253 dimensions, we observed 3 non-circular masses of data (Figure S2A) and, therefore, divided the

254 PETHs into 3 groups. These groups were associated with low, medium, and high changes in

255 spike rate, but had similar activation durations (Figure S2B). Most ensembles (88\%, green and

256 red in Figures S2B and S2C) were characterized by a low or medium change in single unit spike

257 rate corresponding to an increase of 1 to 3 spikes per sec (Figure S2B). In the maximal case,

258 average spike rate increased by 7 spikes per sec (Figure $\mathbf{S 2 B}$, light purple line), but this was the

259 smallest group of ensembles (Figure S2C, light purple). Single unit spike rate for those units

260 within the ensemble was higher when the ensemble was active than when it was inactive (Figure

261 S2D, gray, two-sided Wilcoxon rank sum test, $\mathrm{Z}=20.9, \mathrm{D}=0.8$, power $=0.99, \mathrm{p}<0.001)$. We

262 also assessed the average spike rate when all single units within an ensemble were merged into a

263 single multi-unit spike train. Again, spike rate within the ensemble was higher during epochs of

264 ensemble activation (Figure S2E, gray, two-sided Wilcoxon rank sum test, $Z=14.7, D=2.6$,

265 power $=0.99, \mathrm{p}<0.001)$. On the other hand, when an ensemble was inactive, multi-unit activity

266 outside of the ensemble was relatively higher (Figure S2E, light green, two-sided Wilcoxon rank

267 sum test, $\mathrm{Z}=6.8, \mathrm{D}=0.8$, power $=0.99, \mathrm{p}<0.001)$. This is due to those units spiking as

268 members of other ensembles during these epochs. These results show that the firing strength can

269 vary considerably across LC ensembles. 
bioRxiv preprint doi: https://doi.org/10.1101/2020.03.30.015354; this version posted September 1, 2021. The copyright holder for this preprint (which was not certified by peer review) is the author/funder, who has granted bioRxiv a license to display the preprint in perpetuity. It is made available under aCC-BY-NC-ND 4.0 International license.

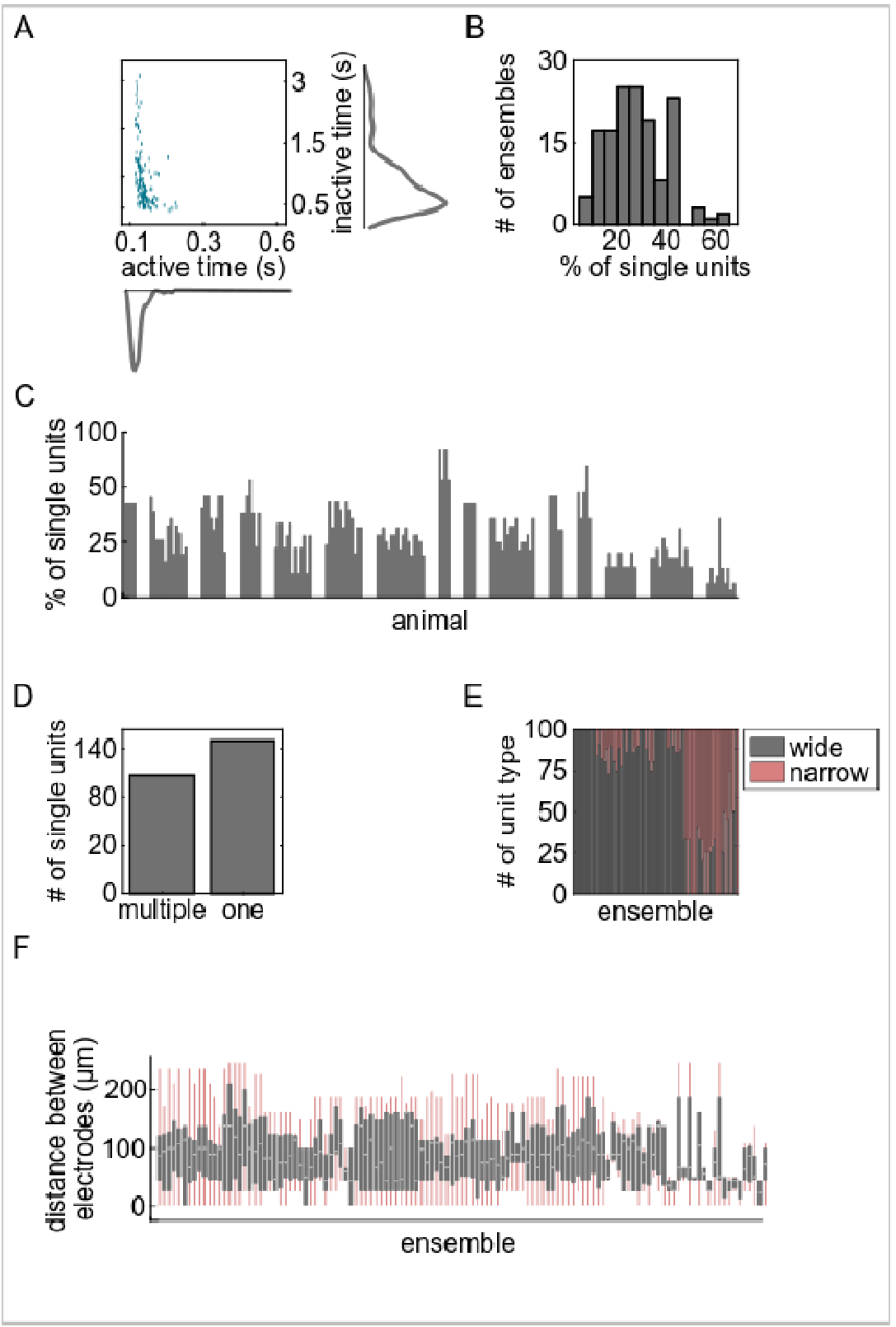


Figure 2. LC ensembles are spatio-temporally sparse and cell type-specific. (A) The scatter plot shows the average ensemble active times versus inactive times along with the corresponding histograms. The narrow distribution of active times indicates that most ensembles are only briefly active $(\sim 100 \mathrm{msec})$ before a longer and more variable period of inactivity that is apparent from the broad distribution of inactive times. (B) The distribution of ensemble sizes is plotted. The values are the percentage of simultaneously recorded single units that were assigned to an ensemble. On average, each ensemble consisted of $27 \%$ of the single units recorded in that experiment. (C) Bar plot showing the percentage of single units simultaneously recorded in each rat that belonged to each ensemble. Each bar reports the percentage of one ensemble. Bars are grouped by rat. Note that a single unit can be part of more than one ensemble. (D) More single units participated in a single ensemble, but many single units were also observed participating in multiple ensembles. (E) The percent of each unit type (wide or narrow spike waveform) making up each ensemble is plotted across ensembles. Rats in which only one single unit type was recorded are not included in this plot. The histogram suggests that ensembles typically consist of one single unit type or at least a majority of one type of unit. (F) Boxplots showing the distribution of the distance among the electrodes from which each pair of single units within each ensemble were recorded. Ensembles with only two single units were excluded. Ensembles are spatially diffuse. time-averaged pairwise correlations used in prior work does not

292 Recent work has demonstrated that most pairs of LC single units do not fire in strong 293 synchronization, but rather show sparse yet structured cross-correlations (20). A graph-theoretic 294 community detection analysis on the pairs with significant time-averaged cross-correlations 295 demonstrated cases in which some single units tended to synchronize their spiking with multiple 296 other single units (20). This analysis is suggestive of a sparse structure of interactions among LC 297 neurons, but it is merely compatible with the possibility that spontaneous LC activity is made up 298 of ensembles. Here, we formally demonstrate that graph theory analysis of time-averaged cross299 correlations, as used in (20), is neither sufficient for identifying ensembles, nor can it determine 300 the times at which distinct ensembles are active. These limitations are illustrated in the following 301 examples, which are also used to demonstrate the power of NMF to detect distinct ensembles 302 each of which has its own unique temporal evolution of activity.

303 We generated three different scenarios of simulated spike trains (Figure 3). Each scenario 304 consisted of 10 simultaneously recorded single neurons that were all governed by ensemble 305 dynamics. In all three scenarios, the graph-theoretic analysis of cross-correlations shown in the 
306 bottom left panels performed exactly as in prior work on LC population activity (20). They 307 revealed the same set of units that were more strongly correlated with each other: units 2 through 3087 and units 9 through 10 (bounded by the green and red boxes that denote the two "communities" 309 identified by the graph theoretic analysis). Information about the temporal activation patterns of 310 these communities cannot be obtained from the graph-theoretic analysis because, as in prior 311 work (20), it uses time-averaged neuronal activity. However, the ground-truth ensemble 312 dynamics were radically different in each scenario. In the first and second scenario (Figure 3A 313 and 3B, respectively), spike trains were generated as unique temporal sequences of activation of 314 the two ensembles (ensemble 1 - units 2 through 7; ensemble 2 - units 9 through 10) that were 315 identified by the graph-theoretic correlation analysis as two communities of neurons strongly 316 correlated with each other. However, the graph-theoretic analysis, being based on time-averaged 317 correlations, could not identify that, in these two scenarios, the ensembles had very different 318 activation dynamics. For instance, in the first scenario (Figure 3A), ensemble 1 was the only 319 strongly active ensemble for the first part of the simulation and ensemble 2 was the only strongly 320 active ensemble for the latter part of the stimulation. Whereas, in the second scenario (Figure 321 3B), not only were the periods of lone strong activation of each of the ensembles different from 322 the first scenario, but there was also a period at the end of the simulation when there was 323 intermediate co-activation of both ensembles. In both cases, NMF can capture the units that are 324 members of each ensemble (i.e., by thresholding the spatial module values) and it can reconstruct 325 the temporal dynamics of each ensemble both when they are active alone as well as when they 326 are co-active. These two scenarios (Figures 3A and 3B) highlight that the time-averaged graph327 theoretic analysis cannot resolve ensemble activation dynamics. Finally, in Figure 3C, we 328 considered a third scenario in which the graph-theoretic analysis generated again two 329 communities identical to those in the first two scenarios (Figure 3A and 3B). Critically, however, 330 there were actually three distinct ground-truth ensembles: (ensemble 1 - units 2 through 4; 331 ensemble 2 - units 5 through 7; and ensemble 3 - units 9 through 10). Ensembles 1 and 2 had 332 different temporal activation patterns with often only one of the two ensembles being active, but 333 they also had a period in which they were both strongly coactive. The graph theoretic analysis 334 based on time-averaged cross-correlations incorrectly conflated the first two ensembles into a 335 single community made of units 2 through 7 . This illustrates that the time-averaged correlation 336 analysis and graph theory detection of communities cannot resolve the identity of individual 
337 ensembles. Thus, our prior work (20), which reported the first large-scale single unit recordings 338 in the LC, successfully demonstrated with the time-averaged correlation analysis that LC 339 populations did not fire en masse, but this was insufficient to demonstrate whether the LC 340 contains ensembles. Furthermore, it could not measure when each ensemble was active.

341 Importantly, we verified that the present NMF analysis was able to detect correctly in all 3 342 scenarios both the identity of the ensembles and the time course of their activation that was used 343 to generate the simulated data (see upper right panels, Figure 3). NMF allowed us to go beyond 344 prior work (20) and assess how LC ensemble dynamics related that of other LC ensembles and, 345 most importantly, to ongoing cortical state dynamics. 


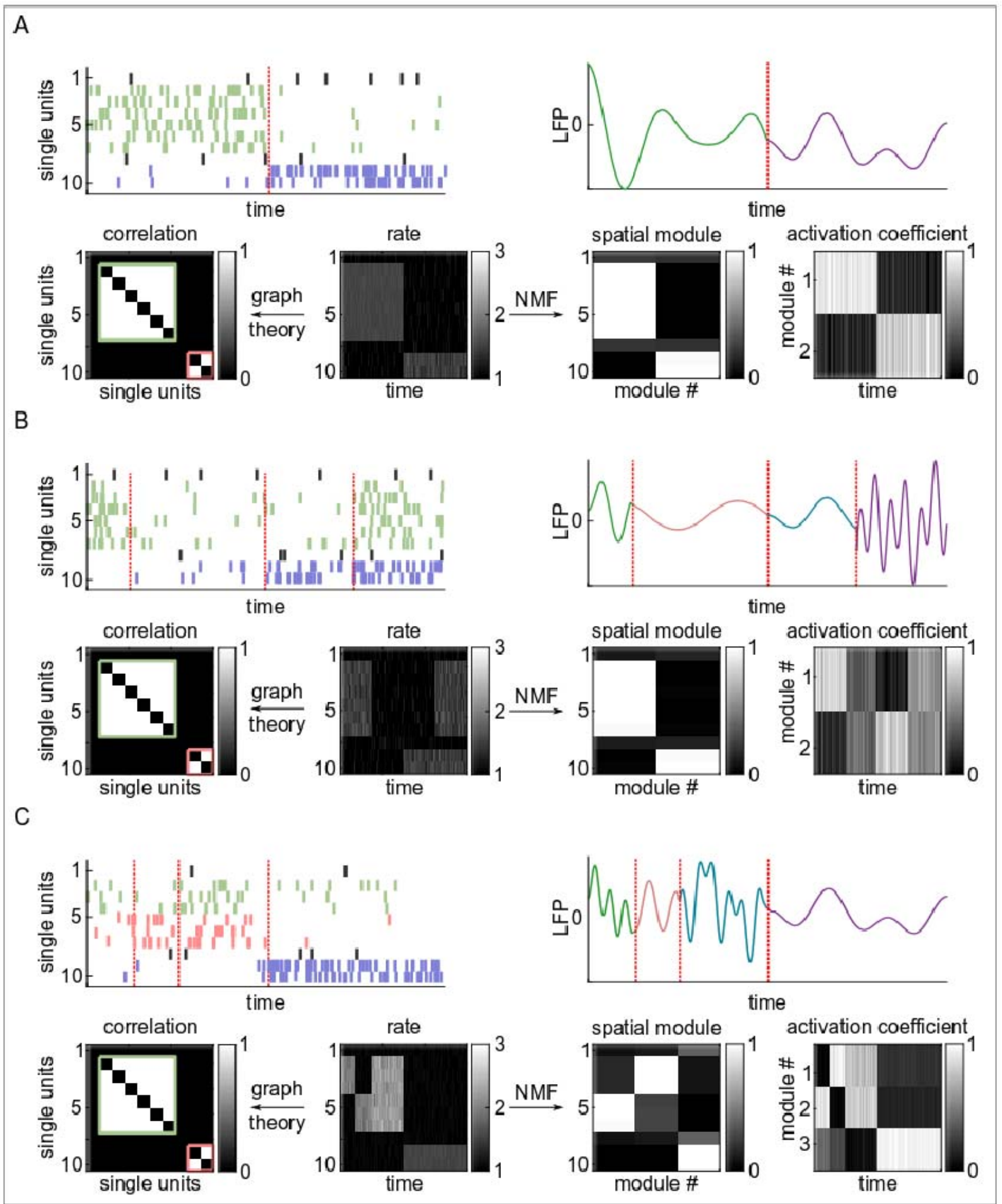

Figure 3. Ensembles cannot be detected using graph-theoretic analysis of time-averaged pairwise correlations. Three different simulated spike rasters were generated in order to compare the NMF method with the graph-theoretic method. In all panels (A-C), the upper left plots depict the generated spike rasters of 10 single units. The spike trains in each panel were generated to have unique ensemble dynamics. The spikes of units belonging to different ensembles are in different colors, while the spikes of units that do not belong to any module are 
black. These ensembles are ground-truths encoded into the simulation. The red dotted lines indicate transitions in the synthesized LC population activity. The same time points are presented on the top right plots, which show the simulated ongoing cortical activity with different cortical states plotted in different colors. In each panel (A-C), the lower panels show the binned spike rates of each unit constructed from the spike rasters (middle), the communities detected by graph theory analysis (left), and the spatial modules (i.e., ensembles) and the ensemble activation time courses (right). In the graph-theoretic analysis, as in prior work (20), the spike count correlation coefficient was calculated from the binned spike counts from the entire recording. Each axis of the lower left plot is a unit and, if the time-averaged correlation for a cell-pair was significant, it was assigned a value of 1 (white) and otherwise 0 (black). Community detection algorithms (as in prior work) were used to detect communities of co-active neurons. These are indicated by the green and red bounding boxes. The result of the NMF analysis (lower right two panels) show the spatial modules and the temporal activation patterns of each ensemble.

Before considering how the activation of LC ensembles relates to cortical states, it is important to further confirm that the NMF-detected LC ensembles are indeed distinct ensembles each with largely non-overlapping activation times, so that different ensembles could potentially produce ensemble-specific cortical states. Our earlier analyses have shown that LC ensembles are activated briefly with long and highly variable duration pauses between one activation and the next (Figure 1C, 2A). This result is highly suggestive that the ensembles are distinct because such pauses can maintain largely non-overlapping activation times between ensembles. Here, we 375 more formally characterized these pauses by examining ensemble auto-correlogram and ensemble-pair cross-correlogram troughs. Using the time series of activations of each LC ensemble, we individuated significant troughs in the ensemble auto-correlograms (Figure 4A)

378 and the ensemble-pair cross-correlograms (Figure 4B). These troughs may be mediated by noradrenergic self-inhibition and lateral inhibition, which is a known property of LC neurons (26-29). A trough in the auto-correlogram, which indicates self-inhibition, occurred in $62 \%$ of the ensembles (90 out of 146). For these ensembles, the self-inhibited spiking was most frequent at a $100 \mathrm{msec}$ delay after ensemble activation but could occur as late as $300 \mathrm{msec}$ after ensemble

383 activation (Figure 4C). In addition to this self-inhibitory property of LC ensembles, we found 384 that $44 \%$ of ensemble-pairs (348 out of 790) had a significant cross-correlogram trough, which 385 indicates a lateral inhibitory interaction. Lateral inhibitory interactions between ensemble-pairs 
were most frequent after a delay of at $\pm 300 \mathrm{msec}$, but covered a wide range of variable timings, lasting up to 1 second (Figure 4D). These times of self-inhibition and lateral inhibition match well the wide range of pauses apparent in ensemble activity shown in Figure $\mathbf{2 A}$ and both types of inhibition likely contribute together to generate these pauses in LC ensemble firing. Overall, our results suggest that these inhibitory mechanisms could help produce the sparse activations of

391 distinct LC ensembles, replete with long and highly variable pauses, such that each LC ensemble 392 has its own unique temporal activity dynamics. Critically, the presence of lateral inhibition 393 between ensembles is also key evidence that ensembles are distinct from one another and, in 394 combination with our simulations showing that NMF can discriminate distinct ensembles 395 (Figure 3), our analysis of ensemble-pair cross-correlograms strongly supports the claim that

NMF can detect distinct ensembles in the LC.

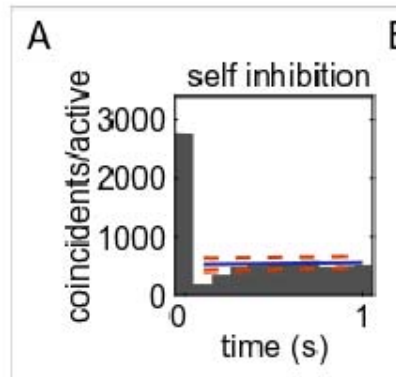

B

C
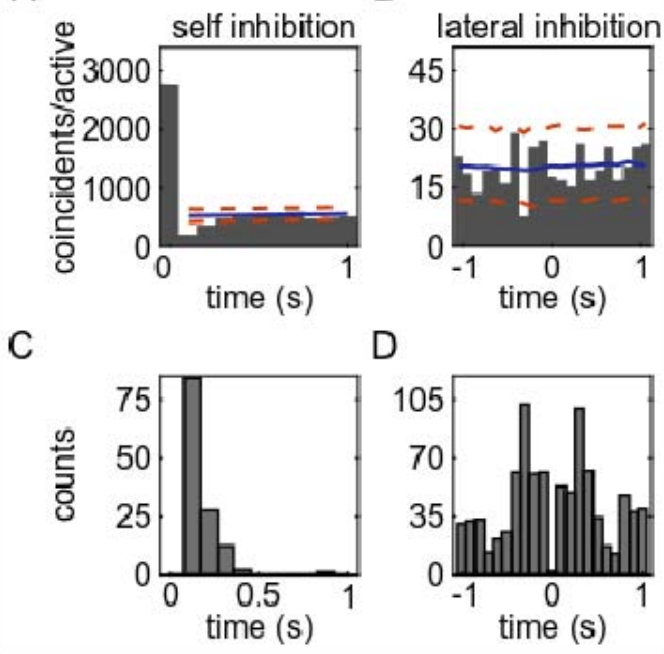

Figure 4. The durations of self-inhibition and lateral inhibition demonstrate that LC ensembles are distinct and their activation pauses occur over 100 milliseconds up to 1 second. (A) An example of a self-inhibition of an ensemble visible as a trough in the autocorrelogram. The example is ensemble \#5 from rat 2591. The time bin is $100 \mathrm{msec}$. Significant troughs were defined as those that crossed the lower bound of the $1 \%$ pairwise minimum threshold (dashed orange lines), which was calculated from 1000 surrogate data sets constructed by jittering ensemble active times. The solid blue line shows the average of the surrogate correlograms. (B) An example of lateral inhibition between an ensemble pair (ensembles 9 and 11 from rat 2632). All details are the same as in panel A. (C) The histogram shows the number of significant self-inhibitions across different time bins. For example, the ensemble shown in panel A had significant troughs at $100 \mathrm{msec}$ and $200 \mathrm{msec}$ after it was activated. Therefore, it all auto-correlogram time points across all ensembles) lasted less than $300 \mathrm{msec}$. (D) The 
411 histogram shows the number of significant lateral inhibitions across different time bins. Lateral 412 inhibition peaked at $\pm 300 \mathrm{msec}$.

\section{Highly synchronous LC population activity occurs, but rarely}

414 Although our results clearly demonstrate that LC ensembles are distinct from one another and 415 activate with largely non-overlapping time courses that contain numerous highly variable firing 416 pauses, it does not preclude the possibility that LC ensembles co-activate. Examples of this in

417 LC data can be seen in Figure 1C (e.g., brown and red lines at $\mathrm{t}=0.5 \mathrm{sec}$ ). The occurrence of 418 ensemble co-activation is important to quantify because such ensemble population activations 419 would be more like the en masse firing evoked in LC stimulation studies that have been used to 420 define the role of the LC in modulating cortical state (10-13).

421 We quantified the number of ensemble-pairs with significant co-activations. We also assessed 422 the most common delay at which one ensemble was activated after the other in the pair because 423 zero-lag pairwise ensemble activations would be like en masse LC activation. Importantly, NMF 424 analysis of synthetic spike trains has demonstrated that this method can detect co-activate 425 ensembles (see Figure 3B). We found that $64 \%$ of 790 ensemble-pairs had positive cross426 correlogram peaks. An example significant positive interaction between two ensembles is shown 427 in Figure 5A. We found that most of the significant positive interactions occurred without a 428 delay (Figure 5B). Specifically, zero-lag (no delay) interaction was observed in $83 \%$ of the $64 \%$ 429 of ensemble-pairs with significant positive interactions. In the overall population of 790 430 ensemble-pairs, this corresponds to synchronous co-activation of $53 \%$ of ensembles. Such zero431 lag positive interactions indicated some degree of co-activation of ensembles. This result shows 432 that highly synchronous and non-delayed co-activation of LC ensembles occurs among a large 433 proportion of ensemble-pairs. These results suggest that, while LC ensemble are distinct, many 434 ensembles co-activate and can therefore mimic the en masse activation of LC neurons due to 435 electrical or optogenetic LC stimulation.

436 To measure how often LC ensembles synchronously co-activate, we used a zero-lag 437 synchronization index, which measures what proportion of activation instances of an ensemble 438 were zero-lag co-activations with another ensemble. The calculation was performed on only the $43953 \%$ of ensemble-pairs with significant zero-lag synchrony. The average synchronization index 
was $28 \%$ (Figure 5B, inset), indicating that the 53\% of ensemble-pairs with significant zero-lag co-activations were synchronously activated only occasionally (i.e., on $28 \%$ of activation

442 instances). Overall, and contrary to the standard view that the LC neuronal population fires en 443 masse with a high level of synchrony, these analyses show that LC ensembles had nuanced and 444 largely non-overlapping dynamics with rare ensemble co-activations.

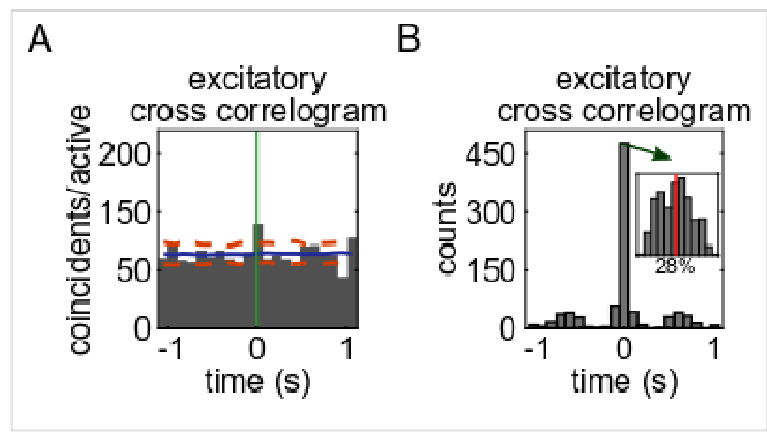

446 Figure 5. Highly synchronous, zero-lag activation happened among $\mathbf{5 3 \%}$ of ensemble-pairs, 447 but its occurrence was rare. (A) An example ensemble-pair with significant positive interaction 448 with zero-lag. The activation time of one ensemble in the pair is at $t=0$ (green line). The example is between ensembles 1 and 2 from rat 1052. The time bin is $100 \mathrm{msec}$. Significant peaks were defined as those that crossed the upper bound of the $1 \%$ pairwise maximum threshold (dashed orange lines). The solid blue line shows the average of the surrogate correlograms. (B) The histogram shows the number of significant ensemble-pair positive interactions across time bins. The majority of positive interactions between ensembles occurs at zero-lag, indicating that many ensemble-pairs synchronously co-activate. The inset presents a histogram of synchrony index values between the ensemble-pairs that had significant zero-lag positive interactions. The average synchrony index is $28 \%$, which indicates that co-activation occurs on only $28 \%$ of ensemble activation instances.

460 The rich, rarely overlapping dynamics of individual LC ensembles may enable distinct 461 ensembles to evoke different cortical states. Alternatively, all ensembles may promote the 462 stereotypical activated state that is widely viewed as the singular role of noradrenergic 463 neuromodulation of cortical state (1-3, 10-13). We disambiguated between these two 464 possibilities using an LC-ensemble-triggered LFP (LCET-LFP) analysis. The ability to examine 
the relationship between LC ensemble activity and ongoing cortical state is enabled by NMF, which for the first time has identified LC ensembles and their temporal activation dynamics.

The LCET-LFP analysis triggered cortical area 24a LFP on the activation of individual LC ensembles. NMF identified 89 ensembles in 9 rats from which cortical LFPs and LC population activity were simultaneously recorded. We calculated the LFP spectrogram modulation in a window of $400 \mathrm{msec}$ before ensemble activation until $500 \mathrm{msec}$ afterwards. This window was chosen for two reasons. First, it provided a good tradeoff between temporal and spectral resolution. Second, our previous analyses of cross-correlations and durations of activation and

473 inactivation show that it is unlikely that multiple ensembles were coactive during this window

474 (Figure 2, Figure 5, and Figure 6). Therefore, this window ensured that changes in the cortical 475 LFP spectrum were predominantly related to activation of an individual ensemble in the LC. We 476 averaged the spectral modulations for each ensemble over all instances its activation. Visual 477 inspection of the LC ensemble activation-triggered spectra revealed diverse cortical states 478 depending on which ensemble was activated (Figure S3).

479 Given this apparent diversity of cortical states associated with the activation times of different 480 LC ensembles, we formally assessed if the multitude of LC ensemble-associated cortical states 481 could be consolidated into a few typical patterns. We clustered the spectral modulations 482 associated with each of the 89 LC ensembles. We found 4 predominant types of spectra in the 483 clustering analysis. We chose 4 clusters by first varying the putative number of clusters from 1 to 48422 and quantifying the diminishing returns of adding each additional cluster (for details, see 485 Methods and Figure S4). Critically, only one of these spectral types (Cluster 1, Figure 6) can be 486 described as the stereotypical "activated" cortical state (10-13). The activated cortical state has 487 been previously characterized in extensive prior work that used electrical or optogenetic 488 stimulation to activate LC neurons en masse and found that this state is a decrease in theta and 489 alpha band oscillations and an increase in gamma band oscillation power of the mean 490 extracellular field potential in both non-anesthetized and anesthetized preparations (10-13). Our 491 analysis revealed that spontaneous activations of LC ensembles in cluster 1 evoked the activated 492 state (Figure 6A). Activation of this sub-set of LC ensembles evoked decreased power in theta 493 and alpha band LFP oscillations and increased power in gamma band oscillations (Figure 6B). 
494 This stereotypical activated cortical state was associated with activation of $28 \%$ of the 89 495 ensembles.

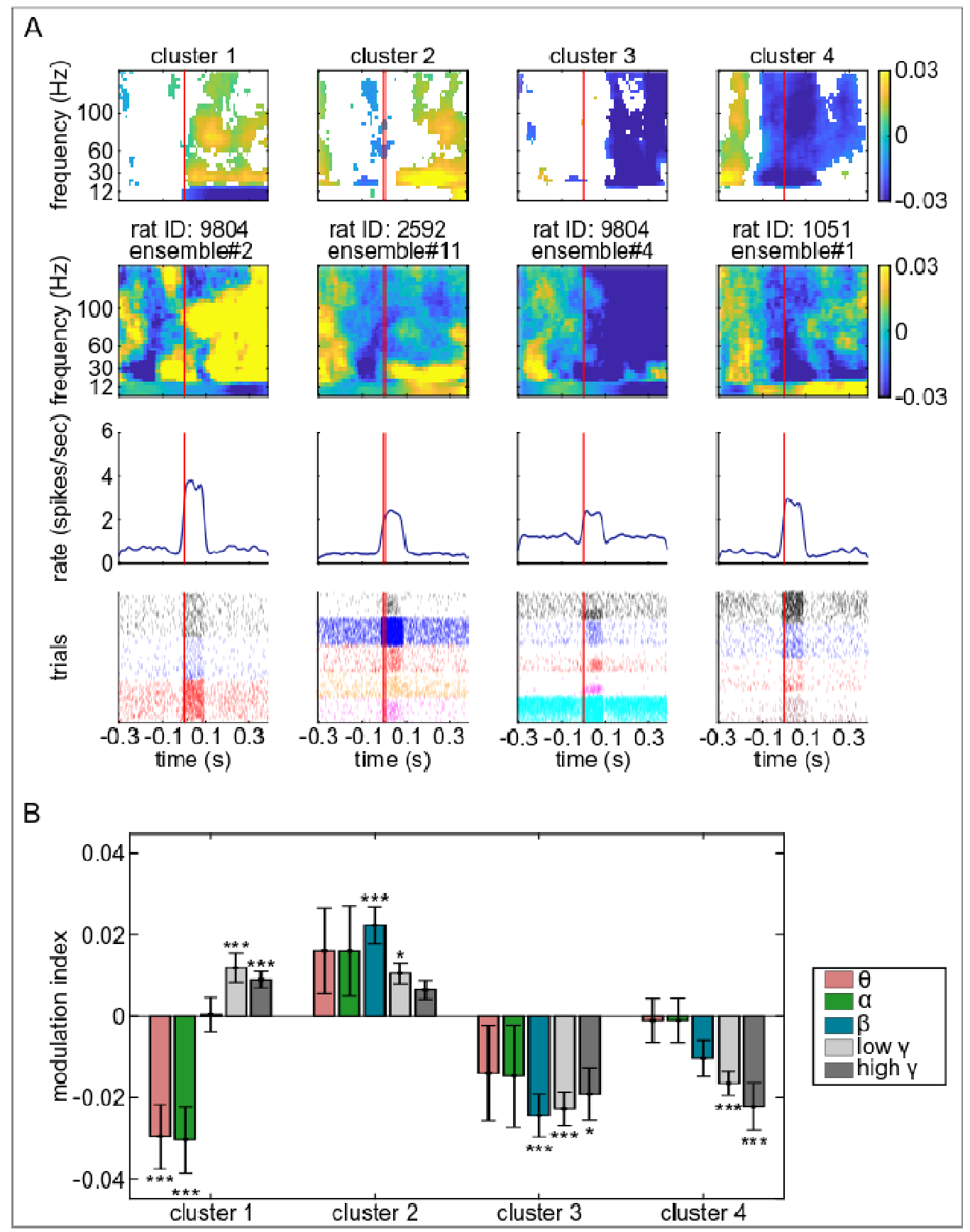

497 Figure 6. Activation of different LC ensembles are associated with diverse changes in 498 cortical LFP power spectra. (A) LFP power spectra were triggered on LC ensemble activation 499 times. The resulting spectra were clustered into 4 types, which are shown as 4 columns. The top 500 row shows the average spectrogram across all spectra in each cluster. Only significant 501 modulations (yellow - increase, blue - decrease) are shown; non-significant values are white. 
The ensemble activation time is at time 0 and is marked by a solid red line. The lower 3 rows show the activity of an example ensemble from each of the 4 spectral clusters and the ensemble activation-triggered spectrogram for that ensemble. The example spectra show both significant and non-significant values. (B) The average modulation indices for different frequency bands are plotted for each cluster. The frequency bands are theta $(4-8 \mathrm{~Hz})$, alpha $(8-12 \mathrm{~Hz})$, beta (12-30 $\mathrm{Hz})$, low gamma $(30-70 \mathrm{~Hz})$ and high gamma $(70-150 \mathrm{~Hz})$. Significant modulations are assessed by testing the median of the modulation distribution against zero by using Wilcoxon's

However, a diverse set of cortical states occurred after activation of different sub-sets of LC ensembles. The second type of ensemble-specific spectra (Cluster 2) was associated with activation of $22.5 \%$ of $\mathrm{LC}$ ensembles and was characterized by a specific increase in beta oscillations and a small increase in low gamma oscillations, whereas power in other frequency bands did not change significantly. The third type of ensemble-specific spectra (Cluster 3) opposed the direction of the first two spectral types, in that the beta, low gamma, and high gamma bands were decreased. This spectral pattern was associated with $22.5 \%$ of the ensembles. In Clusters 1 through 3, the change in cortical state took place after LC ensembles activated. However, the last type of spectrum (Cluster 4) was associated with a change in cortical state that began before LC ensemble activation, namely a decrease in high frequency spectral power. Overall, distinct LC ensembles were associated with spectro-temporally diverse cortical states.

522 Given that the firing strength was variable across LC ensembles (Figure S3), we assessed 523 whether there was a systematic difference in the strength of ensemble population spike rate 524 across the 4 cortical state clusters shown in Figure 6. For instance, the stereotypical activated 525 state (cluster 1 cortical spectra) might only occur when an LC ensemble reaches a certain 526 threshold spike rate. Therefore, we assessed the firing strengths of LC ensembles and whether 527 these differences might predict the relationship between which LC ensembles are associated with 528 each of the 4 different cortical states associated with LC ensemble activation. The population 529 spike rate was calculated as the average of all ensemble activation events combined across all 530 single units in the ensemble (i.e., in the spike rasters shown in Figure 6, all events of different 531 colors were averaged). The peak of the resulting population spike rate was used to characterize 532 the firing strength of the population in each ensemble. We found that the median population 533 spike rate across ensembles in each cortical spectral cluster differed across clusters (Kruskal534 Wallis test, $\left.\mathrm{p}=0.0003, \omega^{2}=0.9633, \chi^{2}=18.82\right)$, but post-hoc tests showed that only cluster 1 
535 was different from clusters 2 and 3; therefore, there was no systematic relationship between

536 cortical spectral cluster type and population spike rate (Figure 7A). We also examined the peak

537 spike rate of the single units in each ensemble. For this analysis, the spike rates around ensemble

538 activation events were first averaged for each single unit separately (i.e., in the spike rasters

539 shown in Figure 6, all events of the same color were first averaged). The peak spike rate of the

540 PETH of each single unit was averaged across units to obtain a measure of single unit firing

541 strength. The median spike rate across all ensembles in each cortical spectral cluster type again

542 differed across clusters (Kruskal-Wallis test, $\mathrm{p}=0.0334, \quad=0.9871, \quad=8.71$ ). The result was

543 similar to that obtained using the population spike rate, in that the single unit firing rate differed

544 only between clusters 1 and 3 (Figure 7B).

545 We also examined another factor that could predict how distinct LC ensembles are associated

546 with different cortical states. Specifically, the size of the ensemble (i.e., the number of single

547 units within the ensemble) might systematically vary with the cortical spectral cluster type. For

548 instance, a type 1 cluster might only be observed when ensembles of a particular size are

549 activated. We assessed this relationship by calculating the median number of units across

550 ensembles in each cortical state cluster. Ensemble size differed across clusters (Kruskal-Wallis

551 test, $\mathrm{p}=0.0029, \quad=0.9608, \quad=13.97$ ), but only between clusters 1 and 3 (Figure 7C). These

552 results demonstrate that, while cluster 1 and 3 differ, there is no systematic relationship between

553 the size of an ensemble and cortical state. Overall, our results demonstrate that cortical state

554 depends on which specific ensembles are active, rather than simply an overall increase in the 555 number of active single units or their firing strength.
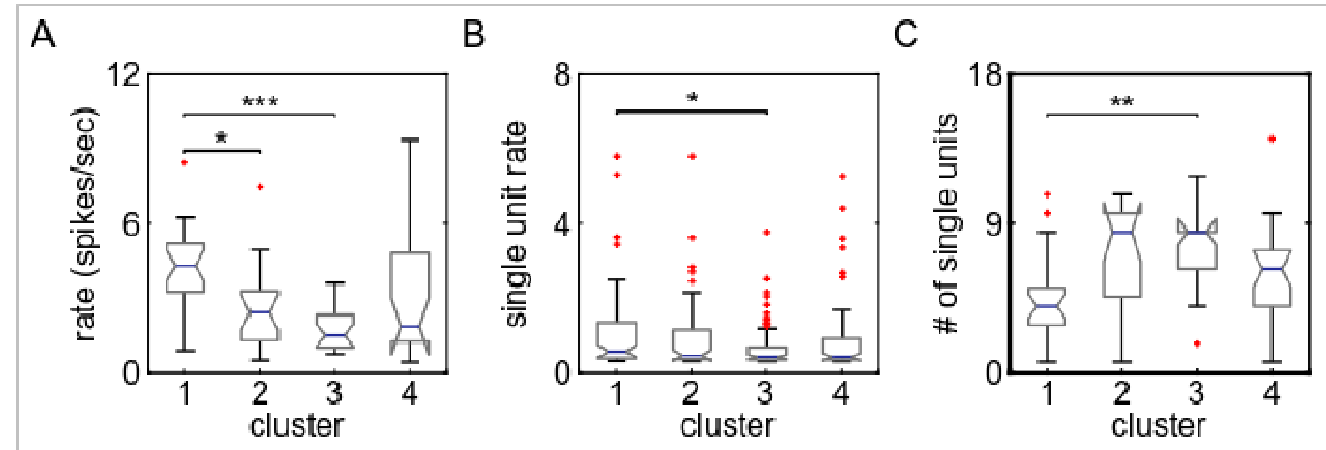
Figure 7. Different cortical states are not related to the intrinsic properties of distinct subsets of LC ensembles. (A) The box plots show the distributions of maximal spike rates of the ensembles' PETHs in each spectral cluster. There was a significant difference in spike rate between clusters 1 and 2, as well as between clusters 1 and 3. (B) The boxplots illustrate the distribution of the spike rate averaged across single units within the ensembles and separating the ensembles by spectral cluster. A significant difference was observed only between clusters 1 and 3. (C) The boxplots show the distributions of the number of single units within the ensembles for the different spectral clusters. A significance difference was found only between spectral clusters

5651 and 3.

\section{Activating a larger pool of LC ensembles results in a more homogeneous cortical state}

568 These data clearly demonstrate a relationship between distinct LC ensembles and different 569 cortical states. This finding stands in marked contrast to the stereotypical activated state evoked 570 by stimulation of the LC, which evokes en masse spiking by LC neurons. Therefore, we 571 predicted that when LC ensembles are co-active (i.e., more of the LC neurons are activated 572 synchronously and LC population activity becomes more similar to stimulation-evoked en masse 573 LC activation), the associated cortical state should become more homogenous to the activated 574 state observed in studies that stimulated the LC. We took advantage of our observation that pairs 575 of LC ensembles can sometimes become co-active (Figure 5B). We assessed the cortical LFP 576 spectra, as in Figure 6, but triggered cortical spectrograms only on coactivation times of 577 ensemble-pairs. A total of 199 ensemble-pairs had a significant zero-lag positive interactions in 578 their cross-correlograms, which indicates co-activation. In contrast with the four heterogenous cortical states observed during activation of individual LC ensembles, k-means clustering now

580 revealed only two types of cortical power spectra at the time points when ensemble-pairs were

581 synchronously co-activated (Figure 8). One cluster is the stereotypical activated cortical state

582 (cluster 2, 103 of 199 ensemble-pairs) and the other cluster is a homogenous decrease in spectral 583 power (cluster 1, 96 of 199 ensemble-pairs). Therefore, when multiple LC ensembles are co584 active, such that LC population activity becomes more similar to en masse LC activation, the 585 modulation of cortical state is more homogenous and similar to LC stimulation-evoked cortical 586 state changes (10-13). 


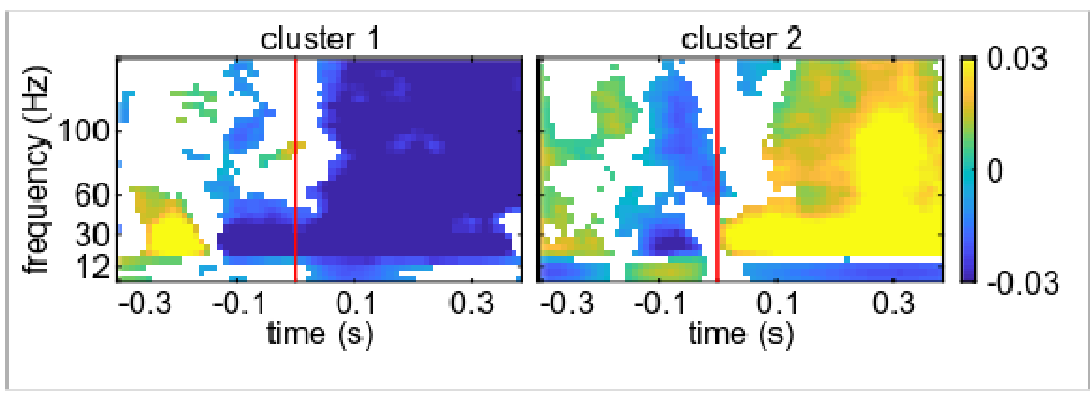

588 Figure 8. Synchronous coactivations of LC ensemble-pairs are associated with more 589 homogeneous changes in cortical LFP power spectra. The LFP power spectra were triggered 590 on the co-activation times of LC ensemble-pairs that had significant zero-lag cross-correlations. 591 The resulting spectra clustered into 2 spectral types. Each plot shows the average spectrogram 592 across all ensemble-pairs associated with each type of spectrum. Only significant changes in the power spectrum are plotted in color; non-significant modulations are white.

\section{Discussion}

Cortical states can vary over a wide range and have been shown to be in a tight relationship with many functions that are relevant to psychiatric disorders, such as sleep, arousal, perceptual ability, and reaction times. It is thus no surprise that there have been long-standing efforts to understand the neural factors contributing to cortical state fluctuations (1-3). However, the selforganized neuronal interactions that control cortical states remain largely elusive. Blocking out the effect of the external world (e.g., slow wave sleep or anesthesia) has proven to be a successful approach for dissecting the spontaneous brain-internal neuronal interactions that control cortical state (30). These approaches have been used to demonstrate that the LC evokes transitions to, and maintenance of, a single and unitary activated state in the cortex (10-13). However, rather than studying the spontaneous emergence of cortical states due to LC activity, these studies have used electrical or optogenetic stimulation of the LC, which evokes en masse LC population activity.

607 Here, we considered the effect of spontaneously occurring events of LC ensemble activations. 608 We demonstrate for the first time that spontaneous LC population activity consists of multiple, 609 discrete neuronal ensembles each with their own nuanced time course of activity by applying $610 \mathrm{NMF}$, an emerging computational methodology. Using synthetic ground-truth spike trains with 611 nuanced patterns of ensemble activity, we illustrate that the time-averaged correlation analysis 
612 and graph theory detection of communities used in prior work (20) cannot resolve the identity of

613 individual ensembles. While the first large-scale single unit recordings in the LC did successfully

614 demonstrate with time-averaged correlation analysis that LC populations did not fire en masse, it

615 was insufficient to demonstrate whether the LC contains ensembles.

616 Using NMF to define the neurons in distinct ensembles and the time courses over which the

617 ensembles were active, we could study for the first time the self-organized spontaneous

618 interactions between LC neurons and cortical states $(25,31)$ and therefore contribute to long-

619 standing efforts to understand the how cortical state might be generated (1-3). The LCET-LFP

620 analysis demonstrated that ongoing cortical state differs depending upon which LC ensemble is

621 activated. Importantly, these findings establish that LC ensembles do not simply evoke a

622 stereotypical activated state in the cortex. Instead, the nuanced, temporally diverse and largely

623 non-overlapping nature of spontaneous LC ensemble activations correspond to a diversity of

624 cortical states. Equally importantly, in the relatively rare cases when LC ensemble-pairs are

625 coactivated synchronously, which is a situation more similar to the highly synchronized and en

626 masse LC population activation driven by LC stimulation (10-13), the diverse set of cortical

627 states collapses toward the stereotypical activated state that has been uniformly observed across

628 these prior studies using LC stimulation.

Potential neurophysiological causes of the diversity in cortical state

630 Neuromodulation of different forebrain regions may alter the self-organized brain-internal 631 neuronal interactions that produce various cortical states. LC neurons are broadly-projecting, but 632 also have localized projections to the forebrain and release a range of neurotransmitters $(32,33)$;

633 therefore, LC ensembles that project to different forebrain neuronal networks could affect how

634 those networks self-organize cortical states. When considering how distinct LC ensembles could 635 promote different cortical states, two potential factors for future study are the diversity of 636 ensemble neurochemical make-up and/or its projection profile. Given that the region in which we 637 assessed cortical state (area 24a) receives projections from approximately 61 to $65 \%$ of LC 638 neurons in the rat $(34,35)$, it seems likely that most of the ensembles project to area $24 \mathrm{a}$ and they 639 should, therefore, produce a similar state change. Our finding to the contrary could be explained 640 by the possibility that the neurochemical make-up of the LC neurons differs across ensembles 
641 and results in cortical state diversity. Another possibility is that, in spite of most ensembles

642 presumably sharing area $24 \mathrm{a}$ as a projection target, it is the other targets that are potentially not

643 shared across ensembles, which leads to LC ensemble-specific cortical states in 24a. According

644 to this forebrain 'network' perspective, LC ensembles associated with different cortical states

645 could have divergent axon collaterals which enable the ensembles to modulate distinct forebrain

646 neuronal networks that are associated with different cortical states.

647 Nuanced activation patterns of diverse LC ensembles enable greater diversity in 648 neuromodulatory functions

649 Behavioral and mental states fluctuate widely from moment-to-moment and it has been known 650 since the advent of EEG recordings that such diverse cognitive-behavioral states are associated 651 with a multitude of cortical states (1-3). The brain-internal interactions that generate this large 652 state-space are still largely unknown. LC neurons were classically thought to modulate cortical 653 and thalamic neuronal excitability level using noradrenergic "tone" (4-7), whereas the neuronal 654 interactions that produce the cortical state are contained within the cortex and thalamus $(36,37)$. 655 According to this standard view, the role of the LC has been to modulate or predispose cortico656 thalamic circuits toward the activated cortical state (and predispose the organism toward 657 wakefulness), but the actual neuronal interactions that select cortical state are between cortex and 658 thalamus. However, this standard view of cortical state generation was developed using methods 659 that artificially activated the LC neuronal population en masse using external stimulation. Here, 660 we used LCET-LFP to study the spontaneously self-organized neuronal interactions which are 661 internal to the brain to reveal that, in contrast to this classical thinking, distinct LC ensembles can 662 promote diverse cortical states. Each ensemble in this a small population of $\sim 1,600$ brainstem 663 noradrenergic neurons may individually be a key player in selecting ongoing cortical state from a 664 multitude of possibilities. Thus, our findings shift the role of the LC from 'modulator / promoter' 665 of a single cortical state toward a 'selector / controller' from a large sub-set of cortical states.

666 Our results imply that a single brainstem nucleus can perform different neuromodulatory 667 functions by simply changing the groups of neurons that fire. Critically, these neuromodulatory 668 dynamics can rapidly change on timescales relevant to flexible and ever-changing cognitive- 
669 behavioral states. The distinct LC ensembles with nuanced activation dynamics shown here

670 substantially enrich the kind of functions that are currently attributed to brainstem nuclei.

\section{Materials and Methods}

672

\section{Recording procedure and signal acquisition.}

674 All experiments were carried out with approval from the local authorities and in compliance with

675 the German Law for the Protection of Animals in experimental research and the European

676 Community Guidelines for the Care and Use of Laboratory Animals. Male Sprague-Dawley rats

677 (350 - 450 g) were used (specific pathogen free, Charles River Laboratories, Sulzfeld, Germany).

678 They were pair housed. Experiments were carried out during the active period of the rats, which

679 were housed on a light cycle of 08:00 to 20:00 darkness. A sub-set of the data were collected

680 from rats used in a prior study (20).

681 Neuronal recordings were made under urethane anesthesia, a widely-used model for studying 682 cortical state transitions evoked by LC stimulation (10, 38). To date, recordings of many LC 683 single units simultaneously in any awake organism with multi-electrode probes has been an 684 intractable problem due to brainstem movement associated with body movement, thus 685 necessitating the use of anesthesia to investigate the relationship between LC ensemble activity 686 and cortical state. Rats were anesthetized using an intra-peritoneal (i.p.) injection of urethane at a 687 dose of $1.5 \mathrm{~g} / \mathrm{kg}$ body weight (Sigma-Aldrich, U2500). Surgical procedures were as described in 688 prior work (20). Electrodes targeted the LC and the prelimbic division of the medial prefrontal 689 cortex. The coordinates for LC were $4.0 \mathrm{~mm}$ posterior from lambda, $1.2 \mathrm{~mm}$ lateral from lambda, 690 and approximately $6.0 \mathrm{~mm}$ ventral from the brain surface (implanted at a 15 deg posterior angle).

691 The coordinates for the cortex were $3.0 \mathrm{~mm}$ anterior and $0.8 \mathrm{~mm}$ lateral from bregma and 3.0 $692 \mathrm{~mm}$ ventral from the brain surface. The LC electrode was targeted based on standard 693 electrophysiological criteria (20). At the end of the recording, we administered clonidine $(0.05$ $694 \mathrm{mg} / \mathrm{kg}$ ) i.p. (Sigma-Aldrich, product identification: C7897) to confirm cessation of noradrenergic 695 neuron spiking. We also verified LC targeting in most experiments using histological 696 examination of coronal sections (50 um thick) that were stained for Cresyl violet or a DAB and 
697 horse radish peroxidase reaction with hydrogen peroxide to visualize an antibody against

698 tyrosine hydroxylase, as shown in prior work (20).

699 The LC was recorded using a multi-channel silicon probe (NeuroNexus, Model: A1x32-Poly3$70010 \mathrm{~mm}-25 \mathrm{~s}-177-\mathrm{A} 32)$. The impedance of the electrodes was $\sim 1.0$ to $2.0 \mathrm{MOhm}$. Cortical local

701 field potentials were recorded using a single tungsten electrode with an impedance of $200-800$

$702 \mathrm{kOhm}$ (FHC). A chlorided silver wire inserted into the neck muscle was used as a ground.

703 Electrodes were connected to a pre-amplifier (in-house constructed) via low noise cables. Analog

704 signals were amplified (by 2000 for LC and 500 for cortex) and filtered (8 kHz low pass, DC

705 high pass) using an Alpha-Omega multi-channel processor (Alpha-Omega, Model: MPC Plus).

706 Signals were then digitized at $24 \mathrm{kHz}$ using a data acquisition device (CED, Model:

707 Power1401mkII).

708 NMF decomposition of population spike trains into coactive ensembles.

709 We used non-negative matrix factorization (NMF) (39) to decompose a matrix of the spike

710 counts of all simultaneously recorded single units across time intervals. NMF linearly

711 decomposes the matrix of the spike counts of the population of single units at each time interval

712 as a sum across a set of non-negative basis functions (modules) using non-negative coefficients

$713(23,24,39)$. The non-negativity constraint is useful for obtaining sparse representations and it is

714 particular suitable for decomposing population spike count at different time intervals, which are

715 always non-negative. Previous work has shown that the NMF of population spike trains provides

716 a robust decomposition whose basis functions can be biologically interpreted as a set of the firing

717 patterns of the single units that are coactive (i.e., an ensemble) and the coefficients quantify the

718 relative strength of recruitment of each ensemble firing pattern at any given time (23).

719 We employed an NMF decomposition that we have previously termed "space only NMF" 720 because it decomposes the population firing patterns across single units at each time interval 721 (23):

$$
\mathrm{R}=\mathrm{WH}+\text { residuals }
$$


$722 \mathrm{R} \in \mathbb{Z}_{+}^{T \times N}$ is the data matrix containing the spike counts of each of $\mathrm{N}$ single units binned into $\mathrm{T}$

723 time bins (with $t$ being the index of each time bin). $\mathrm{H} \in \mathbb{R}_{+}^{K \times N}$ is the matrix containing the basis

724 function, which has $\mathrm{K}$ spatial modules. Each module captures a different pattern of coactivity of

725 the single units and can, therefore, be used to identify which neurons are active together and thus

726 form ensembles. $\mathrm{W} \in \mathbb{R}_{+}^{T \times K}$ is the matrix containing the activation coefficients that describe the

727 strength of recruitment of each module (and thus of each ensemble of coactive neurons) at each

728 time interval. The residuals express the error in the reconstruction of the original population

729 spike train matrix. We computed the decomposition using the multiplicative update rule to

730 minimize the Frobenius norm between the original and the reconstructed data (39). Note that the

731 use of the Frobenius norm assumes that the residuals have a Gaussian white noise structure.

732 One free parameter of the analysis is the temporal resolution of the time binning, $\Delta \mathrm{T}$. We binned

733 spike counts at $\Delta \mathrm{T}=100 \mathrm{msec}$. The time resolution was selected based on our previous work

734 reporting that pairs of LC single units are predominantly synchronized on a timescale of

735 approximately $100 \mathrm{msec}$ or less (20). We also used ranges of $\Delta \mathrm{T}$ from several tens of msec to a

736 few hundreds of msec and found that shorter bins ( $<=20 \mathrm{msec})$ and longer bins ( $>1 \mathrm{~s})$, which our

737 prior work suggests would be outside the range of LC single unit synchrony, tend to artificially

738 identify either many modules each containing only one single active unit or one large ensemble

739 containing all single units, respectively.

740 The second free parameter of the NMF analysis is the number of different modules, $\mathrm{K}$, which 741 were chosen for computing the decomposition. Following established procedures $(23,24)$, we

742 chose $\mathrm{K}$ for each rat by computing the amount of the variance explained by the decomposition

743 when varying $\mathrm{K}$ from its minimum possible value (one) to its maximum possible value (the

744 number of simultaneously-recorded single units). An elbow in this plot indicates a point of

745 diminishing returns for including more modules. We thus chose the number of modules as the

746 smallest $\mathrm{K}$ in the elbow region of this curve for which the decomposition reconstructed at least

$74760 \%$ of the variance of the original spike train data. Given that the NMF decomposition may

748 have local maxima in the variance explained (or equivalently local minima in error

749 reconstruction), after selecting $\mathrm{K}$, we repeated the decomposition five times using this $\mathrm{K}$ and

750 used randomly chosen initialization conditions on each repetition. The selected $\mathrm{K}$ was used if all 
751 solutions had a high degree of stability across these five random initializations. Stability was

752 assessed by checking the repeatability of clustering in comparison to randomly assigning single

753 units to ensembles. The degree of stability was computed as follows. We hard clustered the data

754 to assign each single unit to one and only one ensemble by dividing each column of $\mathrm{H}$ by its

755 maximum and removing the values below 1. From these data we then measured the stability

756 across the five decompositions using the Rand Index (40). We compared the average of the Rand

757 index for each animal with 100 repetitions of the five random clustering. The average Rand

758 Index was always greater than the top 5\% of the distribution of mean Rand Indices resulting

759 from random clustering. Therefore, NMF decomposition produced meaningful and repeatable

760 ensembles. Among those random initializations, the final decomposition reported in the analyses

761 was chosen as the one leading to the maximum variance explained.

762 The modules detected by NMF provide a pattern of coactivation of different single units and the

763 activation coefficients measure the strength of recruitment of each module at any given time.

764 From these data, we used a threshold-crossing of the coefficients to define when ensembles were

765 active and which single units were active in the ensemble. In order to perform the thresholding,

766 we first normalized the columns of $\mathrm{H}$ to the minimum and maximum and then set a threshold

767 based on the distribution of coefficients. Single units within a module were defined as an

768 ensemble of coactive single units if their corresponding element of $\mathrm{H}$ crossed the initial big peak

769 of the histogram of the distribution of coefficient values for that rat (which usually corresponded

770 to $95^{\text {th }}$ percentile). Coefficients below this value were set to zero and values above the threshold

771 were set to one. In the resulting binary version of the matrix, $\mathrm{H}$, a value of 1 represented spatial

772 modules corresponding to a single unit belonging to an ensemble.

773 The columns of the $\mathrm{W}$ matrix correspond to a set of activation coefficients representing the

774 strength of recruitment of each module at any given time interval. We thresholded these

775 continuous values into binary values using the same method explained above for the spatial

776 modules. The binary version of the matrix, $\mathrm{W}$, hereafter referred to as "activation coefficient

777 matrix," was used to determine whether an ensemble is active or not in each time bin. 
779 To assess whether single units within an ensemble tended to cluster on the recording array, we

780 measured the pairwise distance between the units within each ensemble. The location of each

781 unit was assigned to the electrodes on which the maximal waveform was recorded. Euclidian

782 pairwise distances of the units inside each ensemble were calculated.

\section{Spike train simulation}

784 In order to compare the NMF algorithm with previous work that used graph-theoretic community 785 detection of sub-sets of coactive neurons from the time-averaged correlations between LC cell786 pairs (20), we generated three different sets of spike trains. Each set of spike trains had unique 787 ensemble dynamics as a ground-truth. Spike trains were generated using Poisson Process, with 788 time varying rate every $100 \mathrm{msec}$. The baseline rate was randomly sampled from a Gaussian 789 distribution with a mean of 1 and standard deviation of 0.1 . For the periods of coactivation, the 790 rate was increased by a signal to noise ratio sampled from a Gaussian with a mean of 1.5 and 791 standard deviation of 0.1 . After the generation of the spike trains, we binned and counted the 792 spikes every 100 msec. This matrix was fed to NMF for extraction of spatial and temporal 793 modules. Pairwise spike count correlation was calculated using Pearson correlation on the spike 794 count matrix. A graph was made on the correlation matrix with each unit as a node and the links 795 with the nodes was drawn only for significant correlations. The significance of the correlation 796 was assessed by comparing to 1000 surrogate correlations generated by shuffling spike counts 797 randomly in time. The graph was then used as an input for the Louvain community detection as 798 described in prior work (20).

\section{The assignment of single unit types in the ensembles.}

800 Single unit type was defined by waveform duration, as in prior work (20). We determined if 801 single units of the same type were more likely than chance to belong to an ensemble by 802 computing the exact probability of having ensembles of the same single unit type under the null 803 hypothesis of random assignment. These probabilities were computed by the means of repetition 804 of random sampling (assembling) without replacement. The number of units in the sample was 805 fixed to the number of single units in the ensemble. The number of repetitions for each rat was 
806 the number of ensembles that were empirically found by NMF to consist of only one type of

807 single unit.

Calculation of cross-correlograms between pairs of ensembles.

809 Interactions between pairs of ensembles were measured using cross-correlograms between their

810 time-dependent activation coefficients. Cross-correlograms were calculated in a window of 2000

811 msec with a bin size of $100 \mathrm{msec}$. The cross-correlograms were compared to 1000 surrogate

812 cross-correlograms by jittering the activation times uniformly between \pm 1000 msec. Significant

813 excitatory interactions were those that had cross-correlogram bins which crossed the upper $1 \%$ of

814 pairwise coincidental activations observed in the surrogate data.

815 A synchrony index was used to measure the degree of synchrony between the ensemble pairs

816 that show significant zero-lag synchronous activation. We calculated the synchrony index by

817 dividing the number of co-activations of the two ensembles by the sum of total number of 818 activation of each of the ensembles.

Single unit spike rate PETHs and PETH clustering.

820 The Peri-Event Time Histogram (PETH) of the spike times of single units inside and outside of

821 an ensemble were aligned to events (at $\mathrm{t}=0 \mathrm{msec}$ ), which were the ensemble activation times.

822 We examined spike rate during a window from $100 \mathrm{msec}$ before up to $400 \mathrm{msec}$ after the

823 ensemble activation times and used $1 \mathrm{msec}$ bins. For each single unit, we calculated the average

824 spike rate across activation events as though they were different "trials". PETHs were smoothed

825 with a Gaussian kernel (10 msec width). The PETH for each ensemble was obtained by

826 averaging PETHs across all single units that were active in the ensemble.

827 PETH clustering was done in two steps. First, the dimensionality of the original PETHs in time

828 was reduced using the Principle Component Analyses (41). Two dimensions explained more

829 than $95 \%$ of the variance in the original data. After visualizing the data in the 2 dimensions we

830 observed 3 non-circular masses of data. Therefore, we clustered the data in 3 groups using a

831 Gaussian Mixture Model (GMM) (42). The GMM was calculated with 3 repetitions and full 832 covariances. 
834 We investigated the relation between the activation of LC ensembles and cortical state by 835 triggering cortical LFP spectrograms on the timing of ensemble activation events. Spectra were 836 computed using the multitaper method implemented in Chronux toolbox with 3 tapers and time 837 bandwidth product of 5 (43). Short-time Fourier transforms were computed in a 10 msec moving 838 window with a duration of $200 \mathrm{msec}$. The resulting spectral resolution was $\sim 4 \mathrm{~Hz}$ and the 839 temporal resolution was $10 \mathrm{msec}$. We then averaged the resulting event-triggered spectra across 840 all detected activation events separately for each ensemble.

841 From the averaged spectra, we computed an ensemble activation-triggered spectral modulation 842 that characterized the effects of LC ensemble activation on the cortical LFP power spectrum. The 843 spectral modulation was calculated as follows. We first averaged the spectrogram in time at each 844 frequency for the baseline duration (400 $\mathrm{msec}$ before the ensemble being active) and then 845 subtracted the baseline averaged spectrogram from the original spectrogram at each time step 846 and divided by their sum. This quantity varies between -1 to 1 for each time $t$ and frequency $f$ 847 and describes the average change in cortical LFP power around the time of ensemble activation.

\section{Spectrogram clustering.}

849 The set of so obtained ensemble activation-triggered spectral modulations were clustered, in 850 order to assess the diversity of LC ensemble activation-triggered cortical states. The clustering 851 was performed using the k-means algorithm (44). The k-means algorithm requires specifying a 852 choice for the number of possible clusters and for the mathematical function used to compute the 853 distance between the different spectrograms. We tried various definitions of distance functions 854 (Pearson Correlation, Euclidean distance, cosine, and cityblock), and we chose Pearson 855 correlation as distance function because it gave higher averaged silhouette values (45) (i.e., 856 cleaner clustering). We clustered the spectral modulation into $\mathrm{k}=4$ clusters. This number of 857 clusters was selected because it corresponded to the elbow point (defined as the first point in 858 which the error drops below 5\%) of the curve quantifying the normalized clustering error (error 859 divided by the maximum error) as a function of the selected number of clusters. The error in the

$860 \mathrm{k}$-means clustering is computed as sum of the distances of each data point to their respective 
861 cluster centroid. We assessed the significance of the clustered spectral modulations at each time

862 and frequency by pooling the spectral modulations of all ensembles in each cluster by comparing

863 the median of the population at each point against zero using Wilcoxon signed rank test $(5 \%$

864 significance level). The p-values were corrected for multiple comparisons using Benjamini’s \&

865 Hochberg's method for false discovery rate (46).

866 The above analysis was done taking for clustering all spectral modulations obtained in

867 correspondence of a detected activation of one or more ensembles. We performed a further 868 control analyses in which we clustered only the subset of the spectral modulations during 869 coactivation of ensemble-pairs. The clustering procedure for this control analysis was identical to 870 the one reported above but selected a number of clusters (corresponding to the elbow point of the 871 error curve) equal to 2 clusters.

\section{Acknowledgements}

873 This research was supported by the Helsinki Institute of Life Science (NKT) and the Department 874 of Physiology of Cognitive Processes in the Max Planck Institute for Biological Cybernetics (IZ, 875 NKL, NKT). SP was supported by the Fondazione Caritro and by a SFARI explorer grant (Grant 876 no. 602849).

\section{Author Contributions}

878 Conceptualization: NKT, SP; Methodology: NKT, SN, SP; Formal analysis: SN; Investigation:

879 IZ, NKT; Resources: NKL, SP; Writing: NKT, SN, SP; Visualization: SN; Supervision: NKT, 880 SP; Funding acquisition: NKL, NKT, SP.

\section{Declaration of Competing Interests}

883 The authors declare no competing interests.

\section{References}

887 1. K. D. Harris, A. Thiele, Cortical state and attention. Nat Rev Neurosci 12, 509523 (2011). 

Responses. Neuron 87, 11431161 (2015).

890 3. D. A. McCormick, D. B. Nestvogel, B. J. He, Neuromodulation of Brain State and Behavior. 891 Annu Rev Neurosci 43, 1-25 (2020).

892 4. L. W. Swanson, B. K. Hartman, The central adrenergic system. An immunofluorescence study 893 of the location of cell bodies and their efferent connections in the rat utilizing dopamine-beta-

894 hydroxylase as a marker. J Comp Neurol 163, 467505 (1975).

895 5. B. D. Waterhouse, D. J. Woodward, Interaction of norepinephrine with cerebrocortical activity

896 evoked by stimulation of somatosensory afferent pathways in the rat?. Exp Neurol 67, 11-34

897 (1980).

898 6. D. A. McCormick, Neurotransmitter actions in the thalamus and cerebral cortex and their role in neuromodulation of thalamocortical activity. Prog Neurobiol 39, 337-388 (1992).

900 7. D. M. Devilbiss, B. D. Waterhouse, The Effects of Tonic Locus Ceruleus Output on Sensory901 Evoked Responses of Ventral Posterior Medial Thalamic and Barrel Field Cortical Neurons in 902 the Awake Rat. J Neurosci 24, 10773-10785 (2004).

903 8. L. W. Swanson, The locus coeruleus: a cytoarchitectonic, Golgi and immunohistochemical

904 study in the albino rat. Brain Research 110, 3956 (1976).

905 9. R. Grzanna, M. E. Molliver, The locus coeruleus in the rat: An immunohistochemical 906 delineation. Neuroscience 5, 2140 (1980).

907 10. A. Marzo, N. K. Totah, R. M. Neves, N. K. Logothetis, O. Eschenko, Unilateral electrical

908 stimulation of rat locus coeruleus elicits bilateral response of norepinephrine neurons and 909 sustained activation of medial prefrontal cortex. J Neurophysiol 111, 2570-2588 (2014).

910 11. M. Steriade, F. Amzica, A. Nuñez, Cholinergic and noradrenergic modulation of the slow 911 (approximately $0.3 \mathrm{~Hz}$ ) oscillation in neocortical cells. Journal of Neurophysiology 70, 1385 9121400 (1993).

913 12. M. E. Carter, et al., Tuning arousal with optogenetic modulation of locus coeruleus neurons.

914 Nat Neurosci 13, 15261533 (2010).

915 13. H. Hayat, et al., Locus coeruleus norepinephrine activity mediates sensory-evoked

916 awakenings from sleep. Sci Adv 6, eaaz4232 (2020).

917 14. V. A. Alvarez, C. C. Chow, E. J. V. Bockstaele, J. T. Williams, Frequency-dependent

918 synchrony in locus ceruleus: role of electrotonic coupling. Proc National Acad Sci 99, 40324036 919 (2002).

920 15. G. Aston-Jones, F. Bloom, Activity of norepinephrine-containing locus coeruleus neurons in 921 behaving rats anticipates fluctuations in the sleep-waking cycle. J Neurosci 1, 876-886 (1981). 
922 16. G. Aston-Jones, F. E. Bloom, Norepinephrine-containing locus coeruleus neurons in

923 behaving rats exhibit pronounced responses to non-noxious environmental stimuli. J Neurosci

924 Official J Soc Neurosci 1, 887-900 (1981).

925 17. F.-J. Chen, S. J. Sara, Locus coeruleus activation by foot shock or electrical stimulation

926 inhibits amygdala neurons. Neuroscience 144, 472481 (2007).

927 18. P. G. Finlayson, K. C. Marshall, Synchronous bursting of locus coeruleus neurons in tissue

928 culture. Neuroscience 24, 217225 (1988).

929 19. M. Ishimatsu, J. T. Williams, Synchronous Activity in Locus Coeruleus Results from

930 Dendritic Interactions in Pericoerulear Regions. J Neurosci 16, 5196-5204 (1996).

931 20. N. K. Totah, R. M. Neves, S. Panzeri, N. K. Logothetis, O. Eschenko, The Locus Coeruleus

932 Is a Complex and Differentiated Neuromodulatory System. Neuron 99, 1055-1068.e6 (2018).

933 21. S. K. McCune, M. M. Voigt, J. M. Hill\$, Expression of multiple alpha adrenergic receptor

934 subtype messenger RNAs in the adult rat brain. Neuroscience 57, 143-151 (1993).

935 22. G. Paxinos, C. Watson, The Rat Brain in Stereotaxic Coordinates: Compact 7th Edition

936 (Academic Press, 2017).

937 23. A. Onken, et al., Using Matrix and Tensor Factorizations for the Single-Trial Analysis of

938 Population Spike Trains. Plos Comput Biol 12, e1005189 (2016).

939 24. A. H. Williams, et al., Unsupervised Discovery of Demixed, Low-Dimensional Neural

940 Dynamics across Multiple Timescales through Tensor Component Analysis. Neuron 98, 1099-

941 1115.e8 (2018).

942 25. N. K. Logothetis, et al., Hippocampal-cortical interaction during periods of subcortical

943 silence. Nature 491, 547553 (2012).

944 26. G. K. Aghajanian, J. M. Cedarbaum, R. Y. Wang, Evidence for norepinephrine-mediated

945 collateral inhibition of locus coeruleus neurons. Brain Res 136, 570-577 (1977).

946 27. M. Ennis, G. Aston-Jones, Evidence for self- and neighbor-mediated postactivation inhibition

947 of locus coeruleus neurons. Brain Res 374, 299-305 (1986).

948 28. A. Lee, D. L. Rosin, E. J. V. Bockstaele, $\alpha 2$ A-adrenergic receptors in the rat nucleus locus

949 coeruleus: subcellular localization in catecholaminergic dendrites, astrocytes, and presynaptic

950 axon terminals. Brain Res 795, 157-169 (1998).

951 29. H.-P. Huang, et al., Long latency of evoked quantal transmitter release from somata of locus

952 coeruleus neurons in rat pontine slices. Proc National Acad Sci 104, 14011406 (2007).

953 30. B. Haider, D. A. McCormick, Rapid neocortical dynamics: cellular and network

954 mechanisms. Neuron 62, 171189 (2009).

955 31. J. F. Ramirez-Villegas, et al., Coupling of hippocampal theta and ripples with

956 pontogeniculooccipital waves. Nature 589, 96-102 (2021). 
32. N. K. B. Totah, N. K. Logothetis, O. Eschenko, Noradrenergic ensemble-based modulation of cognition over multiple timescales. Brain Res (2018) https:/doi.org/10.1016/j.brainres.2018.12.031.

960 33. D. J. Chandler, et al., Redefining Noradrenergic Neuromodulation of Behavior: Impacts of a 961 Modular Locus Coeruleus Architecture. J Neurosci Official J Soc Neurosci 39, 8239-8249

962 (2019).

963 34. D. J. Chandler, W.-J. Gao, B. D. Waterhouse, Heterogeneous organization of the locus 964 coeruleus projections to prefrontal and motor cortices. Proc National Acad Sci 111, 68166821 965 (2014).

966 35. D. J. Chandler, C. S. Lamperski, B. D. Waterhouse, Identification and distribution of 967 projections from monoaminergic and cholinergic nuclei to functionally differentiated subregions 968 of prefrontal cortex. Brain Res 1522, 3858 (2013).

969 36. Y. A. Hay, et al., Thalamus mediates neocortical Down state transition via GABAB970 receptor-targeting interneurons. Neuron (2021) https:/doi.org/10.1016/j.neuron.2021.06.030.

971 37. M. Steriade, D. McCormick, T. Sejnowski, Thalamocortical oscillations in the sleeping and 972 aroused brain. Science 262, 679-685 (1993).

973 38. R. M. Neves, S. van Keulen, M. Yang, N. K. Logothetis, O. Eschenko, Locus Coeruleus 974 phasic discharge is essential for stimulus-induced gamma oscillations in the prefrontal cortex. $J$ 975 Neurophysiol 119, jn.00552.2017 (2018).

976 39. D. D. Lee, H. S. Seung, Learning the parts of objects by non-negative matrix factorization.

977 Nature 401, 788-791 (1999).

978 40. W. M. Rand, Objective Criteria for the Evaluation of Clustering Methods. J Am Stat Assoc 979 66, $846(1971)$.

980 41. H. Hotelling, Analysis of a complex of statistical variables into principal components. $J$ Educ 981 Psychol 24, 417-441 (1933).

982 42. G. McLachlan, D. Peel, Wiley Series in Probability and Statistics (2000)

983 https:/doi.org/10.1002/0471721182.

984 43. P. Mitra, H. Bokil, Observed Brain Dynamics (Oxford University Press, 2007)

985 https:/doi.org/10.1093/acprof:oso/9780195178081.001.0001.

986 44. D. Arthur, S. Vassilvitskii, k-means++: The Advantages of Careful Seeding. 1027-1035

987 (2007).

988 45. P. J. Rousseeuw, Silhouettes: A graphical aid to the interpretation and validation of cluster 989 analysis. J Comput Appl Math 20, 53-65 (1987).

990 46. Y. Benjamini, Y. Hochberg, Controlling the False Discovery Rate: A Practical and Powerful 991 Approach to Multiple Testing. J Royal Statistical Soc Ser B Methodol 57, 289-300 (1995). 
bioRxiv preprint doi: https://doi.org/10.1101/2020.03.30.015354; this version posted September 1, 2021. The copyright holder for this preprint (which was not certified by peer review) is the author/funder, who has granted bioRxiv a license to display the preprint in perpetuity. It is made available under aCC-BY-NC-ND 4.0 International license.

992

993 


\section{Supplemental Information}

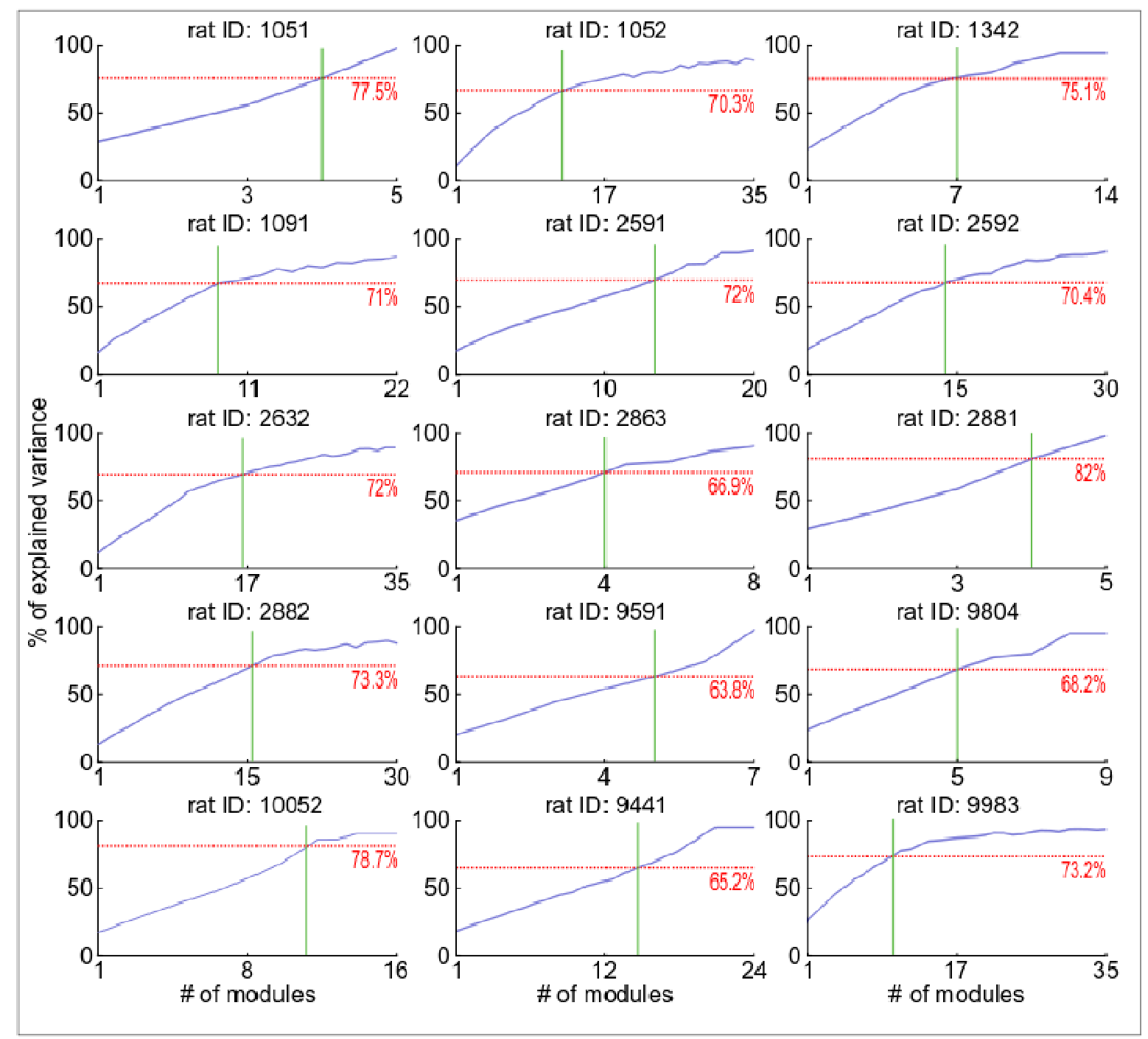

995

996 Figure S1. Data underlying the choice of the optimal number of modules (K) in each rat.

997 Each panel depicts the percentage of explained variance versus the number of the modules for 998 each rat. Solid green lines show the number of selected modules based on the criteria of first 999 elbow after at least $60 \%$ of variance is explained. The dotted red lines show the amount of the 1000 explained variance at the selected number of modules. 


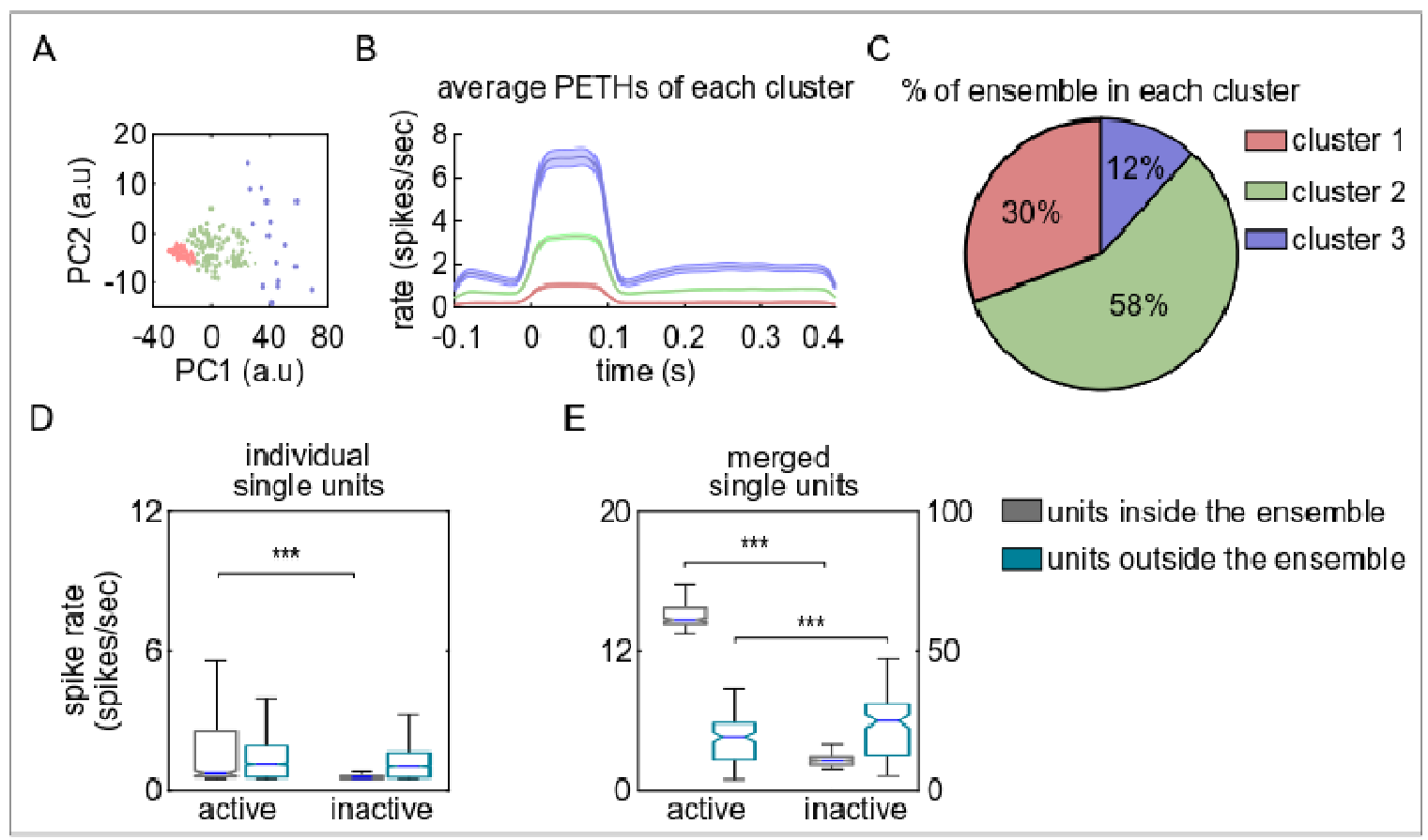

1003 Figure S2. LC ensembles are characterized by different magnitudes of change in spike rate.

1004 (A) The scatter plot shows the projections of the PETHs into two dimensions (PC1, PC2) using 1005 PCA. The first two principal components explained more than $95 \%$ of the variance. Three non1006 circular masses were clustered using GMM. Data points falling into each cluster are color coded 1007 separately. (B) Average PETHs of the ensembles in the same cluster. The zero time on the x-axis 1008 is the ensemble active time. The PETHs of all ensembles were grouped into 3 clusters that 1009 increased their firing rate to different degrees. (C) The pie chart illustrates the percentage of 1010 ensembles in each PETH cluster. Most ensembles had a medium (green) or low (red) magnitude 1011 increase in single unit spike rate. (D, E) The box plots show the distribution of the spike rates for 1012 the single units inside the ensemble (gray) and outside the ensemble (light green). The result is 1013 shown separately for individual single units (C) and the spike trains merged across single units 1014 (D). The spike rate was calculated as the average of all ensemble activation events combined 1015 across all single units in the ensemble (i.e., in Figure 1B and 6A spike rasters, all events of 1016 different colors were averaged). Spike rate increased when the ensemble was in an active state 1017 for both single units and multi-unit activity. Additionally, when the ensemble was inactive, 1018 multi-unit activity outside of the ensemble was higher presumably due to units spiking in other 1019 ensembles. 


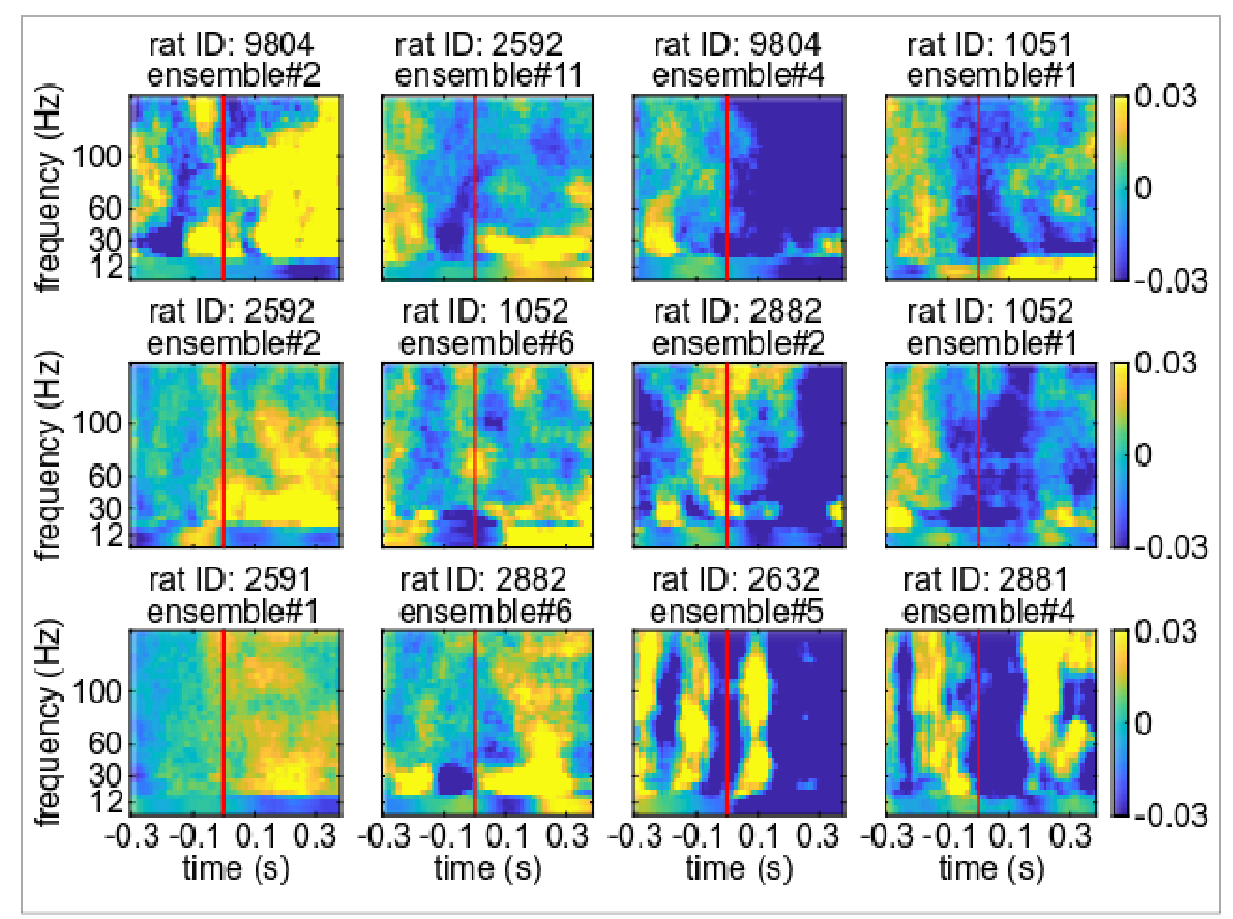

1021 Figure S3. Examples of LC ensemble activation-triggered cortical LFP power spectra. 1022 Examples from 12 different LC ensembles illustrate the diverse cortical states which occur 1023 around the time of ensemble activation. The examples are shown in 4 columns, each of which 1024 indicates a specific trend in the spectra corresponding to the clusters shown in the Figure 5 in the 1025 Main Text. 

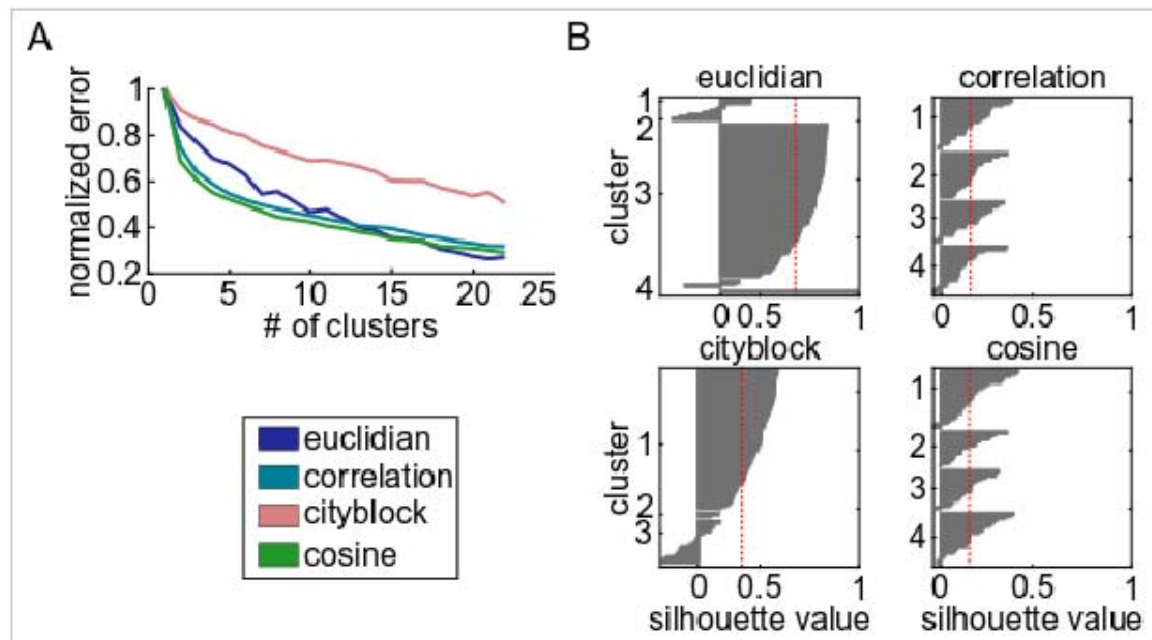

Figure S4. The result of analyses supporting the determination of the best criteria for spectral clustering. (A) The normalized error (error divided by the maximum error) of the kmeans clustering of the ensemble activation-triggered spectra versus the number of clusters. Four different distance measures were assessed and each is plotted in a different color. (B) Each panel shows the result of the silhouette analyses on the chosen number of clusters for four different distance measures. The optimal distance was selected based on both the uniformity in each cluster (the width of the bar plots) and the average silhouette value (the dashed red line). 